\title{
Coincidences in chemical kinetics: surprising news about simple reactions.
}

\author{
G.S. Yablonsky ${ }^{\mathrm{a}, *}$ D. Constales ${ }^{\mathrm{b}, 1}$ G.B. Marin ${ }^{\mathrm{c}}$ \\ ${ }^{a}$ Parks College, Department of Chemistry, Saint Louis University, 3450 Lindell \\ Blvd, Saint Louis, MO 63103, USA. \\ ${ }^{\mathrm{b}}$ Department of Mathematical Analysis, Ghent University, Galglaan 2, B-9000 \\ Gent, Belgium. \\ ${ }^{\mathrm{c}}$ Laboratory for Chemical Technology, Ghent University, Krijgslaan 281 (S5), \\ B-9000 Gent, Belgium.
}

\begin{abstract}
New properties of intersections and coincidences of transient concentration curves were discovered and are presented analytically using the classical consecutive mechanism $A \rightarrow B \rightarrow C$ as an example. We identify six different special points, and analyze and classify the 6 possible (out of 612 combinations) patterns of concentration peak and intersection times and values that distinguish the parameter subdomains and sometimes can eliminate the mechanism. This developed theory is tested on examples (multi-step radioactive decay, isomerization reaction). The mathematical analysis relies on a combination of elementary and symbolic techniques, special functions and numerical approximations.
\end{abstract}

Key words: Kinetic dependence; coincidence; intersection; consecutive mechanism $A \rightarrow B \rightarrow C$; golden ratio; time of intersection.

"Well met, Gentlemen, this is lucky that we meet

so just together at this very door."

- Izaak Walton and Charles Cotton, The Compleat Angler (1676).

* Corresponding author.

Email addresses: gy@che.wustl.edu (G.S. Yablonsky), dcons@world.std.com (D. Constales), Guy.Marin@UGent. be (G.B. Marin).

${ }^{1}$ Financial support from BOF/GOA 01GA0405 of Ghent University is gratefully acknowledged. 


\section{Introduction}

Among the innumerable processes which take place in chemistry and chemical engineering, there are some whose features have as yet been woefully neglected. The goal of the present paper is to distinguish and describe one family of such features, i.e., intersection and coincidences of dependencies which exhibit an unexpected elegance, both physical-chemical and mathematical. Most of the results will be obtained analytically, computer calculations will be used only to solve certain transcendental equations and be mentioned as such.

Consecutive reactions: what was known before

Consecutive reactions are one of the best-known basic mechanisms in chemical kinetics. The simplest example of such sequential reactions is $A \rightarrow B \rightarrow C$. Many important chemical processes are described via this scheme of reactions.

The simplest kinetic model is presented as follows:

$$
\begin{aligned}
\frac{d C_{A}}{d t} & =-k_{1} C_{A}, \\
\frac{d C_{B}}{d t} & =k_{1} C_{A}-k_{2} C_{B}, \\
\frac{d C_{C}}{d t} & =k_{2} C_{B},
\end{aligned}
$$

where $C_{A}, C_{B}, C_{C}$ are the concentrations of the substances $A, B, C$ respectively, and $k_{1}$ and $k_{2}([1 / \mathrm{s}])$ are the rate constants of the first and second reaction, respectively. The temperature dependency of these rate constants is to be of Arrhenius-type,

$$
k_{i}=k_{i, 0} \exp \left(-\frac{E_{A, i}}{R T}\right),
$$

where $k_{i, 0}$ is a pre-exponential factor $[1 / \mathrm{s}], E_{A, i}$ the activation energy $[\mathrm{kJ} / \mathrm{mol}]$, $R$ universal gas constant $[\mathrm{kJ} / \mathrm{mol} \mathrm{K}], T$ is absolute temperature $[\mathrm{K}]$.

The solution of this simple system equations can be found in many popular textbooks (Hlavacek et al., 2007) 37b, (Froment and Bischoff, 1990), on physical chemistry and chemical kinetics, even on Wikipedia. If $C_{C, 0}=0$, the results are well known: when $k_{1} \neq k_{2}$

$$
C_{A}(t)=C_{A, 0} \exp \left(-k_{1} t\right)
$$




$$
\begin{aligned}
C_{B}(t)= & C_{A, 0} k_{1} \frac{\exp \left(-k_{1} t\right)-\exp \left(-k_{2} t\right)}{k_{2}-k_{1}} \\
& +C_{B, 0} \exp \left(-k_{2} t\right), \\
C_{C}(t)= & C_{A, 0}\left[1-\frac{k_{2} \exp \left(-k_{1} t\right)-k_{1} \exp \left(-k_{2} t\right)}{k_{2}-k_{1}}\right] \\
& +C_{B, 0}\left(1-\exp \left(-k_{2} t\right)\right)
\end{aligned}
$$

so that

$$
C_{A}(t)+C_{B}(t)+C_{C}(t)=C_{A, 0}+C_{B, 0}, \quad t \geq 0 .
$$

Otherwise, when $k_{1}=k_{2}$ the system has a different type of solution due to the coincidence of both exponential decays. By direct solution or using the Laplace domain (see Appendix C), it is seen that

$$
\begin{aligned}
& C_{A}(t)=C_{A, 0} \exp \left(-k_{1} t\right), \\
& C_{B}(t)=\left(C_{A, 0} k_{1} t+C_{B, 0}\right) \exp \left(-k_{1} t\right), \\
& C_{C}(t)=C_{A, 0}\left[1-\left(1+k_{1} t\right) \exp \left(-k_{1} t\right)\right]+C_{B, 0}\left(1-\exp \left(-k_{1} t\right)\right) .
\end{aligned}
$$

From here on we assume also $C_{B, 0}=0$. Such formulas can be found e.g. in (Eremin, 1976) and (Bairamov, 2003) p. 49, who presented this solution for $C_{B}(t)$.

Obviously the concentration $C_{B}(t)$ is characterized by a maximum over time, which is a fingerprint of the consecutive scheme $A \rightarrow B \rightarrow C$ contrary to the parallel scheme $A \rightarrow B, A \rightarrow C$. For the latter mechanism, the kinetic model is presented as follows:

$$
\begin{aligned}
\frac{d C_{A}}{d t} & =-\left(k_{1}+k_{2}\right) C_{A}, \\
\frac{d C_{B}}{d t} & =k_{1} C_{A} \\
\frac{d C_{C}}{d t} & =k_{2} C_{A} .
\end{aligned}
$$

The solution of the equations (12)-(14) is

$$
C_{A}(t)=C_{A, 0} \exp \left(-\left(k_{1}+k_{2}\right) t\right)
$$




$$
\begin{aligned}
& C_{B}(t)=C_{A, 0} \frac{k_{1}}{k_{1}+k_{2}}\left[1-\exp \left(-\left(k_{1}+k_{2}\right) t\right)\right], \\
& C_{C}(t)=C_{A, 0} \frac{k_{2}}{k_{1}+k_{2}}\left[1-\exp \left(-\left(k_{1}+k_{2}\right) t\right)\right] .
\end{aligned}
$$

Obviously, in this scheme there is no maximum of any of the concentrations: $C_{A}$ only decreases, $C_{B}$ and $C_{C}$ only increase.

The time of the peak of $C_{B}(t)$ for the consecutive scheme is also presented in the references: from

$$
C_{B}^{\prime}(t)=C_{A, 0} k_{1} \frac{-k_{1} \exp \left(-k_{1} t\right)+k_{2} \exp \left(-k_{2} t\right)}{k_{2}-k_{1}}=0
$$

one solves

$$
t_{B, \max }=\frac{\ln \left(\frac{k_{1}}{k_{2}}\right)}{k_{1}-k_{2}} .
$$

That this satisfies the physicality requirement $t_{B, \max }>0$ follows from the elementary considerations outlined in Appendix A. The maximum value of $C_{B}$ is then given by

$$
C_{B}\left(t_{B, \max }\right)=C_{A, 0}\left(\frac{k_{2}}{k_{1}}\right)^{\frac{k_{2}}{k_{1}-k_{2}}}=C_{A, 0} \rho^{\rho /(1-\rho)},
$$

where we introduce the dimensionless ratio of rate constants $\rho=k_{2} / k_{1}$. See Fig. 1 for the trend of this peak value as a function of $\rho$.

When $k_{1}=k_{2}$, relying or (9)-(11) and equating the time derivative of $C_{B}(t)$ to zero, the maximum of $C_{B}(t)$ occurs at the time

$$
t_{B, \max }=\frac{1}{k_{1}}=\frac{1}{k_{2}} .
$$

and there is a remarkable relationship, which is not widely known:

$$
C_{B}\left(t_{B, \max }\right)=C_{A, 0} e^{-1} .
$$

Studying this consecutive mechanism, we found it independently, but later discovered that it was already mentioned in (Kubasov, 2004). We propose to call this case $k_{1}=k_{2}$ the Euler point $E$. 
To our present knowledge, eqns (1)-(22) comprise all theoretical results obtained for this consecutive scheme. Presently we shall reveal and explain some, to our knowledge as yet unknown, properties of this very simple scheme.

\section{New results on the maximum of $C_{B}$}

\subsection{Concentration patterns and comparison of rate constants}

Calculating the concentration of $A$ at $t_{B, \max }$ from (5) or $(9), C_{A}\left(t_{B, \max }\right)=$ $C_{A, 0} \rho^{1 /(\rho-1)}$ when $\rho \neq 1, C_{A, 0} e^{-1}$ otherwise. Consequently,

$$
\left(C_{B}\left(t_{B, \max }\right) / C_{A, 0}\right)=\left(C_{A}\left(t_{B, \max }\right) / C_{A, 0}\right)^{\rho} .
$$

This is clearly illustrated by the pattern shown on the figures: if $k_{1}=k_{2}$ (as mentioned) the concentrations are equal (Fig. 7); if $k_{1}<k_{2}$ then the concentration of $A$ exceeds that of $B$ (Fig. 9); if $k_{1}>k_{2}$ then the concentration of $B$ exceeds that of $A$ (Fig. 4). Looking at experimental dependencies with these patterns, and assuming the presented consecutive mechanism, we immediately obtain an indication of the comparison between $k_{1}$ and $k_{2}$.

\subsection{Observability of the Euler point}

The question arises whether and if so, when the Euler point is observed, and, more generally, when a given value $\tilde{\rho}$ of $k_{2} / k_{1}$ can be obtained through variation of the temperature $T$. We assume the Arrhenius dependency of the rate coefficients (4). Solving in these terms $k_{2}=\tilde{\rho} k_{1}$ for $T$, we find formally that

$$
T_{\tilde{\rho}}=\frac{1}{R} \frac{E_{A, 1}-E_{A, 2}}{\ln \left(\tilde{\rho} k_{1,0} / k_{2,0}\right)} .
$$

This expression must be positive to represent a physical value of absolute temperature; rewriting the denominator as the difference $\left(\ln \left(\tilde{\rho} k_{1,0}\right)-\ln k_{2,0}\right)$ and again relying on Appendix A, positivity occurs precisely when either both $E_{A, 1}$ and $\tilde{\rho} k_{1,0}$ exceed $E_{A, 2}$ and $k_{2,0}$ respectively, or vice versa.

Therefore, to observe the Euler point $(\tilde{\rho}=1)$ in the working domain of temperatures $100 \mathrm{~K}$ to $500 \mathrm{~K}$ the Arrhenius dependency parameters must meet some limitations, e.g., typically $k_{1,0} / k_{2,0}$ lies between 0.01 to 100 . Then the difference between the activation energies must be less than $20 \mathrm{~kJ} / \mathrm{mol}$ in absolute value. 
If the order of magnitude of the ratio of $k_{1,0} / k_{2,0}$ is 10 , the difference between activation energies has to be of the order of $10 \mathrm{~kJ} / \mathrm{mol}$, which is quite realistic.

\section{Intersections}

\subsection{Intersection of $C_{A}(t)$ and $C_{B}(t)$ curves}

Two types of trajectories are studied: (a) phase trajectories in the space of all variables (concentrations), (b) temporal trajectories of each variable separately. It is a well-known mathematical fact that phase trajectories do not intersect or merge. But the temporal trajectories may or may not intersect. Physico-chemically, the intersection of the temporal concentration curves $C_{A}(t)$ and $C_{B}(t)$, for instance, means that these concentrations can be considered equal, $C_{A}(t)=C_{B}(t)$.

In these subsections, we investigate what kind of temporal concentration intersections can occur under which conditions. The point of this intersection analysis is to classify which orderings can prevail among their characteristics, i.e., the times and values of their occurrences.

A special case of intersection is osculation, in which not only the concentrations but also their temporal slopes coincide, $C_{A}(t)=C_{B}(t)$ and $C_{A}^{\prime}(t)=C_{B}^{\prime}(t)$. Generally, it is possible to classify different qualitative cases:

(1) there is a single intersection at finite time, e.g., Fig. 4;

(2) there are multiple non-osculating intersections, e.g., Fig. 2;

(3) there is at least one osculating intersection, e.g., Fig. 3;

(4) there is no intersection, e.g., Fig. 10;

(5) intersection is achieved at time $+\infty$, e.g., Fig. 9.

We now have from solving $C_{A}(t)=C_{B}(t)$ from (5) and (6) for $t$ that

$$
t_{A=B}=\frac{\ln \left(\frac{k_{1}}{2 k_{1}-k_{2}}\right)}{k_{2}-k_{1}}
$$

and distinguish the following cases:

(1) when $k_{2}<2 k_{1}$ and $k_{1} \neq k_{2}$, the logarithm is taken of a positive value, and rewriting the expression as

$$
t_{A=B}=\frac{\ln k_{1}-\ln \left(2 k_{1}-k_{2}\right)}{k_{1}-\left(2 k_{1}-k_{2}\right)},
$$


Appendix A clearly shows that it is always a positive value, hence physical. The value at the intersection is

$$
C_{A}\left(t_{A=B}\right)=C_{B}\left(t_{A=B}\right)=C_{A, 0}(2-\rho)^{1 /(\rho-1)}, \quad \rho>0, \quad \rho \neq 1 .
$$

As $k_{2} \rightarrow 0, t_{A=B}$ tends to $\ln (2) / k_{1}$ and $C_{A}\left(t_{A=B}\right)$ to $C_{A, 0} / 2 ;$ as $k_{2} \rightarrow$ $2 k_{1}, t_{A=B}$ tends to infinity and $C_{A}\left(t_{A=B}\right)$ to 0 . See e.g. Fig. 4 .

When $k_{2}=k_{1}$ (Euler point), the equations (9)-(11) must be used instead of (5) and (6). There is also a single intersection, at the time

$$
t_{A=B}=\frac{1}{k_{1}}=\frac{1}{k_{2}}=t_{B, \max }
$$

and

$$
C_{A}\left(t_{A=B}\right)=C_{B}\left(t_{A=B}\right)=e^{-1} .
$$

See e.g. Fig. 7.

(2) There is no intersection when $k_{2}>2 k_{1}$, because the solution to $C_{A}(t)=$ $C_{B}(t)$ then becomes complex. See e.g. Fig. 10.

(3) It is impossible to have multiple non-osculating intersections because there can only be one solution to $C_{A}(t)=C_{B}(t)$, viz $(26)$.

(4) It is impossible for an osculating intersection to occur because the system $C_{A}(t)=C_{B}(t), C_{A}^{\prime}(t)=C_{B}^{\prime}(t)$ has no solutions.

(5) When $k_{2}=2 k_{1}$, intersection is achieved at time $t=+\infty$. See Fig. 9. We shall call this in the subsequent classification the Osculation point.

This analysis can be used to test the hypothesis about the type of mechanism: if more than one intersection of the concentration curves $C_{A}$ and $C_{B}$ is observed, it implies that the consecutive scheme $A \rightarrow B \rightarrow C$ cannot be valid, and a more complex mechanism has to be proposed. If, on the other hand, we assume this consecutive mechanism, the observed fact of intersection of $C_{A}$ and $C_{B}$ immediately gives us a restriction on the domain of parameters: $k_{2}<2 k_{1}$.

\subsection{Intersections of $C_{A}(t)$ and $C_{C}(t)$, and of $C_{B}(t)$ and $C_{C}(t)$.}

As mentioned in the previous subsection, $C_{A}(t)$ and $C_{B}(t)$ do not always intersect; but $C_{A}(t)$ and $C_{C}(t)$, and $C_{B}(t)$ and $C_{C}(t)$ always do; these are therefore called unavoidable intersections.

For the intersection of $C_{A}$ and $C_{C}$ the normalized concentration $C_{A} / C_{A, 0}$ solves as $0<x<1$ the following equation:

$$
x^{\rho}+x(1-2 \rho)+(\rho-1)=0,
$$


when $\rho \neq 1$, otherwise

$$
x \ln x-2 x+1=0,
$$

whose solution is $x=\exp \left(2+W_{-1}\left(-e^{2}\right)\right)=0.317844 \ldots$ (see Appendix B for the definition of the Lambert function $\left.W_{-1}\right)$.

For the intersection of $C_{B}$ and $C_{C}$ the normalized concentration $C_{A} / C_{A, 0}$ solves as $x$ the equation

$$
2 x^{\rho}-x(1+\rho)+(\rho-1)=0 .
$$

when $\rho \neq 1$, otherwise

$$
2 x \ln x-x+1=0,
$$

whose solution is $x=\exp \left(\frac{1}{2}+W_{-1}\left(-\frac{1}{2 \sqrt{e}}\right)\right)=0.284668 \ldots$

When $\rho \neq 1$ is a sufficiently simple integer or fraction, equations (30) and (32) can be solved exactly.

In all cases the time of intersection is readily obtained as $(\ln (1 / x)) / k_{1}$ in view of (5) and (9).

\subsection{Intersections of $C_{A}^{\prime}(t)$ and $C_{B}^{\prime}(t)$, and of $C_{B}^{\prime}(t)$ and $C_{C}^{\prime}(t)$}

Solving the equation for $C_{A}^{\prime}(t)=C_{B}^{\prime}(t)$ with respect to $t$, we obtain when $k_{1} \neq k_{2}$

$$
t_{A^{\prime}=B^{\prime}}=\frac{\ln \frac{k_{2}}{2 k_{1}-k_{2}}}{k_{2}-k_{1}},
$$

which is well defined whenever $k_{2}<2 k_{1}$, i.e., $\rho<2$; it is seen to be positive by Appendix A when rewriting it as

$$
t_{A^{\prime}=B^{\prime}}=2 \frac{\ln k_{2}-\ln \left(2 k_{1}-k_{2}\right)}{k_{2}-\left(2 k_{1}-k_{2}\right)},
$$

and relying on Appendix A. At the Euler point the value is $2 / k_{1}$. We then have the remarkable property that

$$
t_{A^{\prime}=B^{\prime}}=t_{B, \max }+t_{A=B} .
$$


On the other hand, solving the equation for $C_{B}^{\prime}(t)=C_{C}^{\prime}(t)$ with respect to $t$, we obtain for $k_{1} \neq k_{2}$

$$
t_{B^{\prime}=C^{\prime}}=\frac{\ln \frac{2 k_{2}}{k_{1}+k_{2}}}{k_{2}-k_{1}},
$$

which is always well defined, and positive as seen from rewriting it as

$$
t_{B^{\prime}=C^{\prime}}=\frac{\ln \left(2 k_{2}\right)-\ln \left(k_{1}+k_{2}\right)}{\left(2 k_{2}\right)-\left(k_{2}+k_{1}\right)}
$$

in view of Appendix A. At the Euler point the value is $1 /\left(2 k_{1}\right)$.

\section{Special points}

In the course of our analysis, we have discovered several special points with characteristic properties, which we will accordingly name and present systematically. For simplicity we assume $C_{A, 0}=1$ here.

\subsection{Acme point, $\rho=1 / 2$}

The Acme point is defined by $k_{2}=k_{1} / 2$, see Fig. 4 . It has the following remarkable intersection and culmination properties:

(1) setting $\rho=1 / 2$ in (30), we find $C_{A}$ and $C_{C}$ cut with $C_{A}=C_{C}=1 / 4=$ $0.25, C_{B}=1 / 2=0.5$ at $t_{A=C}=\ln 4 / k_{1}=1.386294 / k_{1}$;

(2) note that at $t_{A=C}, C_{B}$ culminates (we call this an equiculmination property), and this culmination value is exactly $1 / 2$;

(3) from (25), we see that $C_{A}$ and $C_{B}$ cut with $C_{A}=C_{B}=4 / 9=0.444444$, $C_{C}=1 / 9=0.111111$ at $t_{A=B}=\ln (9 / 4) / k_{1}=0.810930 / k_{1}$;

(4) setting $\rho=1 / 2$ in (32), $C_{B}$ and $C_{C}$ cut with $C_{A}=1 / 9=0.111111$, $C_{B}=C_{C}=4 / 9=0.444444$ at $t_{B=C}=\ln 9 / k_{1}=2.197224 / k_{1}$. This is also the time $t_{A^{\prime}=B^{\prime}}$, and hence the upward slope of $C_{C}$ at this point equals twice the downward slope of $C_{B}$.

(5) There is a remarkable additivity property for the times of intersection: $t_{A=B}+t_{A=C}=t_{B=C}$.

(6) Finally, another value coincidence occurs between the intersections, as $C_{A}\left(t_{A=B}\right)=C_{B}\left(t_{B=C}\right)=4 / 9=0.444444$. 
At the Acme point, the solutions (5)-(7) for $C_{A, 0}=1, C_{B, 0}=0$ can be rewritten (taking into account $k_{2}=k_{1} / 2$ ) as

$$
\begin{aligned}
& C_{A}=\exp \left(-k_{1} t\right)=\left(\sqrt{C_{A}}\right)^{2}, \\
& C_{B}=2 \sqrt{C_{A}}\left(1-\sqrt{C_{A}}\right), \\
& C_{C}=\left(1-\sqrt{C_{A}}\right)^{2}
\end{aligned}
$$

at all times $t$. From this it is clear that for every $\left(C_{A}, C_{B}, C_{C}\right)$ there is also a trajectory point $\left(C_{C}, C_{B}, C_{A}\right)$ (replacing $\sqrt{C_{A}}$ by $\left(1-\sqrt{C_{A}}\right)$ ), i.e., the phase portrait of the trajectory is symmetrical under the exchange of $A$ and $C$. Consequently, if we were to base time on $C_{B}$ (instead of $C_{A}$ or $C_{C}$ as would be more usual), each $C_{B}$ value determines two real times $t_{1}$ and $t_{2}$, so that $\left(C_{A}\left(t_{1}\right), C_{B}\left(t_{1}\right), C_{C}\left(t_{1}\right)\right)=\left(C_{C}\left(t_{2}\right), C_{B}\left(t_{2}\right), C_{A}\left(t_{2}\right)\right)$.

\subsection{Euler point, $\rho=1$}

See Fig. 7.

(1) From (25), $C_{A}$ and $C_{B}$ cut with $C_{A}=C_{B}=1 / e=0.367879$ (which is also the culmination value of $\left.C_{B}\right), C_{C}=1-2 / e=0.264241$ at $t_{A=B}=1 / k_{1}$. (Since $k_{1} C_{A}=k_{2} C_{B}$ necessarily at the maximum of $C_{B}$, from $k_{1}=k_{2}$ it followed immediately $C_{A}=C_{B}$.)

(2) Since $\rho=k_{2} / k_{1}=1$, (31) must be used, and can still be solved in closed form using the Lambert function $W_{-1}: C_{A}$ and $C_{C}$ cut with $C_{A}=C_{C}=$ $\exp \left(2+W_{-1}\left(-e^{-2}\right)\right)=0.317844, C_{B}=1-2 \exp \left(2+W_{-1}\left(-e^{-2}\right)\right)=$ 0.364311 at $t_{A=C}=1.146193 / k_{1}$.

(3) Since $\rho=1,(33)$ applies, which is also solvable in terms of $W_{-1}$, so that $C_{B}$ and $C_{C}$ cut with

$$
C_{A}=\exp \left(1 / 2+W_{-1}(-\exp (1 / 2) / 2)\right)
$$

i.e., $0.284668, C_{B}=C_{C}=0.357665$ at $t_{B=C}=-\left(1 / 2+W_{-1}(-\exp (1 / 2) / 2)\right) / k_{1}=$ $1.256431 / k_{1}$.

\subsection{Lambert point, triple intersection, $\rho=1.1739824 \ldots$}

Is it possible to have a triple intersection, i.e., all three concentrations equal at the same time? It turns out that the condition for this is (see Appendix B 
for the definition of the Lambert function $W_{0}$ )

$$
\frac{k_{2}}{k_{1}}=2-\frac{W_{0}(\ln (1 / 3) / 3)}{\ln (1 / 3)}=1.1739824 \ldots
$$

which we shall denote by $\rho_{L}$, the Lambert point ratio.

At this point, $C_{A}=C_{B}=1 / 3$, so that in view of eqns (5) and (6), and writing $\rho_{L}$ for the solution ratio $k_{2} / k_{1}$

$$
\frac{1}{3}=\frac{1}{\rho_{L}-1}\left(\frac{1}{3}-\left(\frac{1}{3}\right)^{\rho_{L}}\right)
$$

Multiplying by $\left(\rho_{L}-1\right)$ to remove the denominator (at the risk of introducing a parasitic solution $\rho_{L}=1$ ),

$$
\rho_{L}-2+3 \exp \left(\rho_{L} \ln (1 / 3)\right)=0
$$

Now introducing $\alpha$ through $\rho_{L}=2-\alpha / \ln (1 / 3)$, the equation simplifies to $\alpha \exp (\alpha)=(1 / 3) \ln (1 / 3)$. This has two solutions: $\alpha=\ln (1 / 3)$ leads to $\rho=1$, which is parasitic (we know that triple intersection does not occur at the Euler point), and $\alpha=W_{0}((1 / 3) \ln (1 / 3))=-0.907473$, which leads to the value $\rho_{L}$ in (40).

(1) $C_{A}, C_{B}$ and $C_{C}$ intersect simultaneously at value $1 / 3$, time $\ln (3) / k_{1}=$ 1.098612 .

(2) The culmination value of $C_{B}$ is 0.338800 .

The concentrations are plotted in Fig. 8.

\subsection{Osculation point, $\rho=2$}

In what follows, $\phi$ denotes the golden ratio, $\phi=(\sqrt{5}+1) / 2=1.618033 \ldots$

(1) $C_{B}$ culminates at the value $1 / 4$.

(2) $C_{B}$ and $C_{C}$ cut with $C_{B}=C_{C}=1 / 4=0.25, C_{A}=1 / 2=0.5$, at time $t_{B=C}=\ln 2 / k_{1}=0.693147 / k_{1}$.

(3) $C_{A}$ and $C_{C}$ cut with $C_{A}=C_{C}=1 / \phi^{2}=2-\phi=0.381966, C_{B}=2 \phi-3=$ 0.236067 at time $t_{A=C}=2 \ln \phi / k_{1}=0.962423 / k_{1}$.

(4) $C_{A}$ and $C_{B}$ cut at time infinity and value zero (osculation).

Experimentally, we are better able to observe peak and intersection values than osculation at infinity, because tail analysis can be marred by experimental error. 


\subsection{Golden point, $\rho=1 / \phi$}

We can also consider the angle of intersection between concentration curves, but this only makes sense when both axes are commensurate, as can be obtained for instance by replacing time by $C_{C}(t)$. The angle $\alpha(t)$ between the horizontal axis and the tangent to the $C_{A}$ vs. $C_{C}$ curve satisfies at any time $t$,

$$
\tan \alpha(t)=\frac{C_{A}^{\prime}(t)}{C_{C}^{\prime}(t)} .
$$

Similarly, the angle $\beta(t)$ between the horizontal axis and the tangent to the $C_{B}$ vs. $C_{C}$ curve satisfies at any time $t$,

$$
\tan \beta(t)=\frac{C_{B}^{\prime}(t)}{C_{C}^{\prime}(t)} .
$$

Consequently, if $t$ is the time of intersection, then the angle of intersection $\gamma$ is given by their difference, and invoking the formula for the tangent of a difference,

$$
\tan (\alpha-\beta)=\frac{\tan \alpha-\tan \beta}{1+\tan \alpha \tan \beta}
$$

we see that

$$
\tan \gamma=\frac{\left(C_{A}^{\prime}-C_{B}^{\prime}\right) / C_{C}^{\prime}}{1+\left(C_{A}^{\prime} C_{B}^{\prime} / C_{C}^{\prime 2}\right)} .
$$

Simplification then leads to

$$
\tan \gamma=\left|\frac{\rho(\rho-2)}{\rho^{2}+\rho-1}\right|
$$

where we have added the absolute value signs to obtain a geometrically meaningful angle between 0 and 90 degrees.

When this tangent value is infinite, orthogonal intersection occurs, i.e., when $\rho=(\sqrt{5}-1) / 2=1 / \phi=0.618034$, the inverse of the golden ratio. This we call the golden point, see Figs. 5 and 6 . It has the following additional properties:

(1) The culmination value of $C_{B}$ is then $\phi^{-\phi}=0.459040$.

(2) $C_{A}$ and $C_{B}$ cut with $C_{A}=C_{B}=(3-\phi)^{-\phi^{2}}=0.42871, C_{C}=1-2(3-$ $\phi)^{-\phi^{2}}=0.142561$ at $t_{A=B}=\phi^{2} \ln (3-\phi) / k_{1}=0.846952 / k_{1}$; 
(3) $C_{A}$ and $C_{C}$ cut with $C_{A}=C_{C}=0.270633, C_{B}=0.458732$ at $t_{A=C}=$ $1.306989 / k_{1}$

(4) $C_{B}$ and $C_{C}$ cut with $C_{A}=0.155992, C_{B}=C_{C}=0.422003$ at $t_{B=C}=$ $1.857946 / k_{1}$.

Equation (47) also leads to two other interesting special cases: an angle of 45 degrees is associated with the Euler point $\rho=1$ and with another point, $\rho=1 / 3$. Similarly, an angle of 60 degrees occurs for

$$
\begin{aligned}
& \rho=\frac{\sqrt{3} \sqrt{19}+\sqrt{19}-3 \sqrt{3}-5}{4}=0.428145 \\
& \rho=\frac{\sqrt{3} \sqrt{19}-\sqrt{19}+3 \sqrt{3}-5}{4}=0.846771
\end{aligned}
$$

and an angle of 30 degrees for

$$
\begin{aligned}
& \rho=\frac{-\sqrt{3} \sqrt{17}-\sqrt{17}+3 \sqrt{3}+7}{4}=0.232904 \\
& \rho=\frac{\sqrt{3} \sqrt{17}-\sqrt{17}-3 \sqrt{3}+7}{4}=1.205542 .
\end{aligned}
$$

\subsection{Triad point, $\rho=3$}

This point is defined by $k_{2}=3 k_{1}$, see Fig. 10 .

(1) $C_{A}$ and $C_{B}$ do not cut at all.

(2) $C_{A}$ and $C_{C}$ cut with $C_{A}=C_{C}=\sqrt{2}-1=0.414213, C_{B}=3-2 \sqrt{2}=$ 0.171572 at time $t_{A=C}=\ln (\sqrt{2}+1)=0.881373$.

(3) $C_{B}$ and $C_{C}$ cut with $C_{B}=C_{C}=1-\phi / 2=0.190983, C_{A}=1 / \phi=\phi-1=$ 0.618033 , at time $t_{B=C}=\ln \phi / k_{1}=0.481211 / k_{1}$.

(4) $C_{B}$ culminates at the value $\sqrt{3} / 9=0.192450$.

\section{Ordering properties of time and of values for $C_{B, \max }$ and at in- tersections}

\subsection{Properties of maximum and intersection time curves}

In Fig. 11 we plot the values of the time at which the maximum of $C_{B}$ is reached, and those of the time of intersection of $C_{A}, C_{B}$, and $C_{C}$, again with respect to $\rho=k_{2} / k_{1}$, and assuming $k_{1}=1 \mathrm{~s}^{-1}$. 
(1) at the Acme point $A$ the equiculmination implies that the $C_{B, \max }$ and $C_{A}=C_{C}$ curves intersect (simply).

(2) At the Euler point $E$, the $C_{B, \max }$ curve intersects simply with the $C_{A}=$ $C_{B}$ curve.

(3) At the Lambert point $L$, the three intersection curves intersect simply.

(4) At the Osculation point $O$, the $C_{B, \max }$ curve intersects simply with the $C_{B}=C_{C}$ curve, and the $C_{A}=C_{B}$ curves has an asymptote. The practical consequence of this, is that the $C_{C}$ curve intersects the $C_{B}$ curve to the right of its maximum, if and only if the $C_{A}$ and $C_{B}$ curves intersect.

Qualitatively, we can summarize this in Table 1. The result of this analysis is a set of possible patterns for the times at which intersections and the peak occur. Confronting the experimental data with this list of possibilities, we may conclude that the kinetic model relating to the proposed scheme is valid or not.

\subsection{Properties of concentration value curves at maximum and intersections}

In Fig. 12 we plot the values of the maximum of $C_{B}$ and of the intersections between $C_{A}, C_{B}$ and $C_{C}$ with respect to $\rho=k_{2} / k_{1}$. These curves exhibit remarkable properties:

(1) the $C_{A}=C_{B}$ curve and the $C_{B}=C_{C}$ curve intersect simply at the Acme point $A$ (as mentioned there),

(2) the $C_{B, \max }$ and $C_{A}=C_{B}$ curves intersect with osculation at the Euler point $E$.

(3) The $C_{A}=C_{B}, C_{B}=C_{C}$ and $C_{A}=C_{C}$ curves intersect simply at the Lambert point $L$.

(4) The $C_{A}=C_{C}$ and $C_{B \text {,max }}$ intersect simply at the unique value $\rho=k_{2} / k_{1}$ that satisfies the transcendental equation

$$
\rho^{\rho /(\rho-1)}+\frac{1-\rho^{-\rho}}{1-\rho}=2
$$

numerically, $\rho=1.197669$. $C_{B, \max }$ is then 0.335243 , reached at time $0.912520 / k_{1}$, and $C_{A}=C_{C}$ is reached at time $1.092898 / k_{1}$.

(5) The $C_{B, \max }$ and $C_{B}=C_{C}$ curves intersect with osculation at the Osculation point $O$.

Qualitatively, we can summarize this in Table 2. Similarly, the result of this analysis is a set of possible patterns for the values at which intersections and the peak occur. Note that the same value sequence is obtained for the intervals $1 / 2<\rho<1$ and $1<\rho<1.17 \ldots$ this is due to the osculation at the Euler point of the $C_{A}=C_{B}$ and $C_{B, \max }$ curves. 
Tables 1 and 2 present all possible scenarios of observed characteristics of transient behavior in terms of the times of intersection and their values. There are six possible regimes, related to the different parameter domains of $\rho=$ $k_{2} / k_{1}$. The domains are denoted D1 up to D5, the time sequences T1 up to T5, and the value sequences V1 up to V5. Every domain is characterized by two specific sequences of intersection times and values, the relation between which is not always one to one. T1 is related to V1 (see Fig. 13); T2 to V2 (Fig. 14); T3 to V2 as well (Fig. 15); T3 related to V3 or V4, the domain D4 being split in two subdomains D4a and D4b corresponding to V3 and V4, respectively (Figs. 16 and 17). Finally, T5 is related to V5. (Fig. 18). In fact, $\mathrm{D} 4 \mathrm{a}$ is only a tiny domain ( $2 \%$ relative size), which may be hard to observe. Only these scenarios can be observed. The total amount of possible permutations of times and of values is $24+6=30$ each (24 if AB occurs, 6 otherwise), there are $24 \times 24+6 \times 6=612$ joint possibilities, of which surprisingly only 6 can occur.

\subsection{Classification of observable phenomena}

In table 3, A stands for Acme, L stands for Lambert, E for Euler, G for Golden Ratio, O for Osculation, $\mathrm{T}$ for triad.

Concerning the possibility to observe these points in practice: since they are defined by values $\tilde{\rho}$ ranging from $1 / 2$ to $3,(24)$ implies that the considerations for the Euler point $\tilde{\rho}=0$ will carry through on the level of orders of magnitude for the ratios of $k_{i, 0}$ and the difference in activation energy.

\subsection{Right and Wrong in textbooks concerning two-step consecutive reactions}

The formulas presented in textbooks, papers, Wikipedia, for consecutive reactions are correct. In some textbooks, expressions for $t_{B, \max }$ are given. However, no classification and understanding is given of special points and the regimes and scenarios of behavior governed by them. Consequently, the illustrations are sometimes wrong, being impossible in this case. For instance in (Bairamov, 2003), in Fig. 2.1a p. 49 the correct sequence $\mathrm{Bm}<\mathrm{BC}<\mathrm{AC}<\mathrm{AB}$ is exhibited (i.e., between the Lambert and Osculation points, 1.17 . $<\rho<2$, but in Fig. $2.2 \mathrm{a}$ p. 51 , the impossible sequence $\mathrm{BC}<\mathrm{Bm}<\mathrm{AC}<\mathrm{AB}$ is shown.

On the Web, at http://www.meteo.mcgill.ca/233/chap10/sld013.htm, we see another impossible sequence, $\mathrm{Bm}<\mathrm{BC}<\mathrm{AC}$.

Furthermore, in some cases the illustrations unintentionally do correspond to special points, especially the Euler and Acme points, e.g., (Froment and 
Bischoff, 1990), p. 16 (Euler point) and p. 367 (Acme point).

The mistake most often found, however, is to identify the domination of the second exponent as a "quasi-steady-state". Many authors do not understand that there are only two extreme cases of dominating in this reaction: dominating of the first or the second exponent, see eq. (6), and the other exponent has nothing to do with quasi-steady-state regime. These two extreme regimes are presented on Figs. 23 and 24. The first regime, in which $k_{1}$ is very large compared to $k_{2}$, can be considered as the $B \rightarrow C$ regime, the second regime, in which $k_{2}$ is very large, as $A \rightarrow C$.

\section{Selected Applications}

In this section we present selected application to interesting examples.

\subsection{Multi-step radioactive decay}

In such multi-step processes, every step is by definition a first-order process. We can apply our analysis: for instance (see (Lederer et al., 1968)), in the Actinium series, ${ }^{211} \mathrm{Bi}$ alpha-decays to ${ }^{207} \mathrm{Tl}$, this beta-decays to ${ }^{207} \mathrm{~Pb}$ with respective half lifes 2.14 and $4.77 \mathrm{~min}$, respectively. Therefore in this two-step consecutive process, the constant $k_{2} / k_{1}=2.14 / 4.77=0.449$, close to the Acme point.

Similarly, in the Radium series, ${ }^{214} \mathrm{~Pb}$ beta-decays to ${ }^{214} \mathrm{Bi}$, which beta-decays (for $99.98 \%$ ) to ${ }^{214} \mathrm{Po}$, which alpha-decays very rapidly (half-life $0.16 \mathrm{~ms}$ to ${ }^{201} \mathrm{~Pb}$. This multistep decay can be closely approximated by two steps, the first with half-life $27 \mathrm{~min}$, the second $20 \mathrm{~min}$. The corresponding ratio of rate constants is $k_{2} / k_{1}=27 / 20=1.35$, which lies between the Lambert and the Osculation points.

\subsection{Isomerization reactions}

In accordance with data presented by (Wei and Prater, 1962), four interesting cases are considered here and plotted in Figs. 19, 20, 21, 22. 


\section{Experimental error}

When investigating in practice a reactor set-up, measurements will be affected by some level of experimental error.

- For instance, determining the intersection between two curves may be done through linear interpolation by first identifying two data points $\left(t_{1}, C_{1}\right)$ and $\left(t_{2}, C_{2}\right)$ on the first curve, and two $\left(t_{1}^{\prime}, C_{1}^{\prime}\right)$ and $\left(t_{2}^{\prime}, C_{2}^{\prime}\right)$ on the second, such that the two line segments defined by these will intersect. This intersection is then given by $t=D_{t} / D_{1}$ and $C=-D_{C} / D_{1}$, where

$$
D_{1}=\left|\begin{array}{cccccc}
t_{1} & t_{2} & 0 & 0 & 1 & 0 \\
C_{1} & C_{2} & 0 & 0 & 0 & 1 \\
1 & 1 & 0 & 0 & 0 & 0 \\
0 & 0 & t_{1}^{\prime} & t_{2}^{\prime} & 1 & 0 \\
0 & 0 & C_{1}^{\prime} & C_{2}^{\prime} & 0 & 1 \\
0 & 0 & 1 & 1 & 0 & 0
\end{array}\right|
$$

and

$$
D_{t}=\left|\begin{array}{cccccc}
t_{1} & t_{2} & 0 & 0 & 0 & 0 \\
C_{1} & C_{2} & 0 & 0 & 1 & 0 \\
1 & 1 & 0 & 0 & 0 & 1 \\
0 & 0 & t_{1}^{\prime} & t_{2}^{\prime} & 0 & 0 \\
0 & 0 & C_{1}^{\prime} & C_{2}^{\prime} & 1 & 0 \\
0 & 0 & 1 & 1 & 0 & 1
\end{array}\right|, \quad D_{C}=\left|\begin{array}{cccccc}
t_{1} & t_{2} & 0 & 0 & 1 & 0 \\
C_{1} & C_{2} & 0 & 0 & 0 & 0 \\
1 & 1 & 0 & 0 & 0 & 1 \\
0 & 0 & t_{1}^{\prime} & t_{2}^{\prime} & 1 & 0 \\
0 & 0 & C_{1}^{\prime} & C_{2}^{\prime} & 0 & 0 \\
0 & 0 & 1 & 1 & 0 & 1
\end{array}\right| .
$$

Since determinants are multilinear, it is a straightforward application of error analysis to propagate estimates on the time and concentration errors through these formulas, whether using a statistical or an interval approach.

- When a maximum must be located, experimental errors can lead to false values or obscure the true one; typically, one would then fit a least-squares parabola through enough data points and estimate the maximum from that. Again, the mathematics for the resulting formulas is an application of linear algebra techniques.

- More generally, one may wish to use maximally the information provided by all the data points: this can readily be done by fitting the exact solution via the parameters $k_{1}$ and $k_{2}$ to the data, ideally taking into account a full statistical description including repeat experiments, estimated (co)variances, 
etc. If the resulting residues show no persistence of systematic errors (a desideratum that may require substantial correction efforts), the nonlinear least squares method will yield aggregate estimates of the standard deviations $\sigma_{1,2}$ and correlation $r$ on the rate constants values, which can then be propagated through all formulas to determine, e.g., confidence intervals for the identification of special points. In particular, to the ratio $\rho=k_{2} / k_{1}$ there corresponds a variance

$$
\sigma_{\rho}^{2}=\frac{k_{1}^{2} \sigma_{2}^{2}+k_{2}^{2} \sigma_{1}^{2}-2 r k_{1} k_{2} \sigma_{1} \sigma_{2}}{k_{1}^{4}} .
$$

\section{Results and Conclusions}

The analysis that we presented significantly improves our ability to distinguish mechanisms; previously, we considered that observing a maximum of $C_{B}$ is a sufficient indication to distinguish the consecutive mechanism $A \rightarrow B \rightarrow C$ from the parallel $A \rightarrow B, A \rightarrow C$. Now we are able based on our six possible scenarios, either to falsify the hypothesis of the $A \rightarrow B \rightarrow C$ consecutive mechanism (and propose a more complicated alternative), or to justify it and infer some conditions on the ratio $\rho$ of the rate constants.

\subsection{Mathematical technique}

The results of this paper were obtained by careful combinations of elementary and symbolic mathematics, special functions and numerical approximations. We fully intend to use the same array of tools in investigating more complex linear and even some nonlinear models along the same lines.

\subsection{Direct application and future development}

In the nearest future, we will continue analyzing the following series of firstorder reaction mechanisms: parallel irreversible first-order reactions $A \rightarrow B$, $A \rightarrow C$; consecutive first-order reactions in which one step is reversible; triangle of first-order reactions (reversible or not), changing parameters of the system, especially the initial composition and the temperature. Some simple models with nonlinear dependencies will be analyzed as well. The results obtained for the change of concentration in time can be immediately applied to steady-state plug-flow reactor models, in which space or residence time play the role of the astronomic time. Such analyses can also be done for CSTR reactors. 
We will analyze such models both for close systems and for open systems with mass exchange. An interesting opportunity of these are provided by thermodesorption and thermogravimetry problems: in both cases, there is a sequence of linear processes and the kinetic model reflects this sequence and the change of other factor(s) as well. In the first case, the change of temperature in time; in the second, the loss of weight over time. The problem of intersection can be presented for exit flow dependencies for diffusion-reaction models (related to the Temporal Analysis of Products, TAP, data), etc. Special attention will be paid to the analysis of multi-step radioactive decay, where the coefficients are known very accurately.

As the systems considered grow more complex, the number of intersection and maximum points and of their orderings will grow rapidly: polynomially for the former, combinatorially for the latter. This increasing complexity can to some extent be matched by automatic methods, whether numerical and symbolic depending on what the theoretical setting will allow. Ultimately, we expect that unifying properties will emerge; indeed, we generally believe that the analysis of intersections and coincidences presents a source of vast information for new families of patterns for distinguishing mechanisms and parameter domains, and might even find applications outside the domain of chemical kinetics.

\section{List of Symbols}

$A, B, C$ Substances involved in a reaction mechanism.

$C_{A}, C_{B}, C_{C}$ Concentrations of the substances $A, B, C$.

$C_{A, 0}, C_{B, 0}, C_{C, 0}$ Initial values of the substance concentrations. $C_{C, 0}$ is always assumed $0 ; C_{B, 0}$ is assumed 0 in most of the treatment. In the figures $C_{A, 0}$ is conventionally set to 1 .

$E_{A, 1}, E_{A, 2}$ Arrhenius activation energies for $k_{1}$ and $k_{2},[\mathrm{~kJ} / \mathrm{mol}]$; see (4).

$k_{1}, k_{2}$ Reaction rate constants [1/s].

$k_{1,0}, k_{2,0}$ Arrhenius coefficients for $k_{1}$ and $k_{2}$, [1/s[]; see (4).

$\mathcal{L}$ Laplace transform, defined as

$$
\mathcal{L} F(s)=\int_{0}^{\infty} e^{-s t} F(t) d t .
$$

$\phi$ The golden ratio, $(\sqrt{5}+1) / 2=1.618033 \ldots$

$R$ universal gas constant $[\mathrm{kJ} / \mathrm{mol} \mathrm{K}]$.

$\rho$ The dimensionless ratio $k_{2} / k_{1}$. When varying the temperature $T$ to reach a value of $\rho$, the target is denoted $\tilde{\rho}$.

$T$ Absolute temperature $[\mathrm{K}]$.

$t$ Time [s]. 
$W_{0}, W_{-1}$ Principal and $(-1)$ branch of the Lambert $W$-function, see Appendix B. 


\section{List of table captions}

Table 1

Qualitative list of possible cases (time).

Table 2

Qualitative list of possible cases (values).

Table 3

Classification of observable phenomena. 
List of figure captions (in the body of the manuscript)

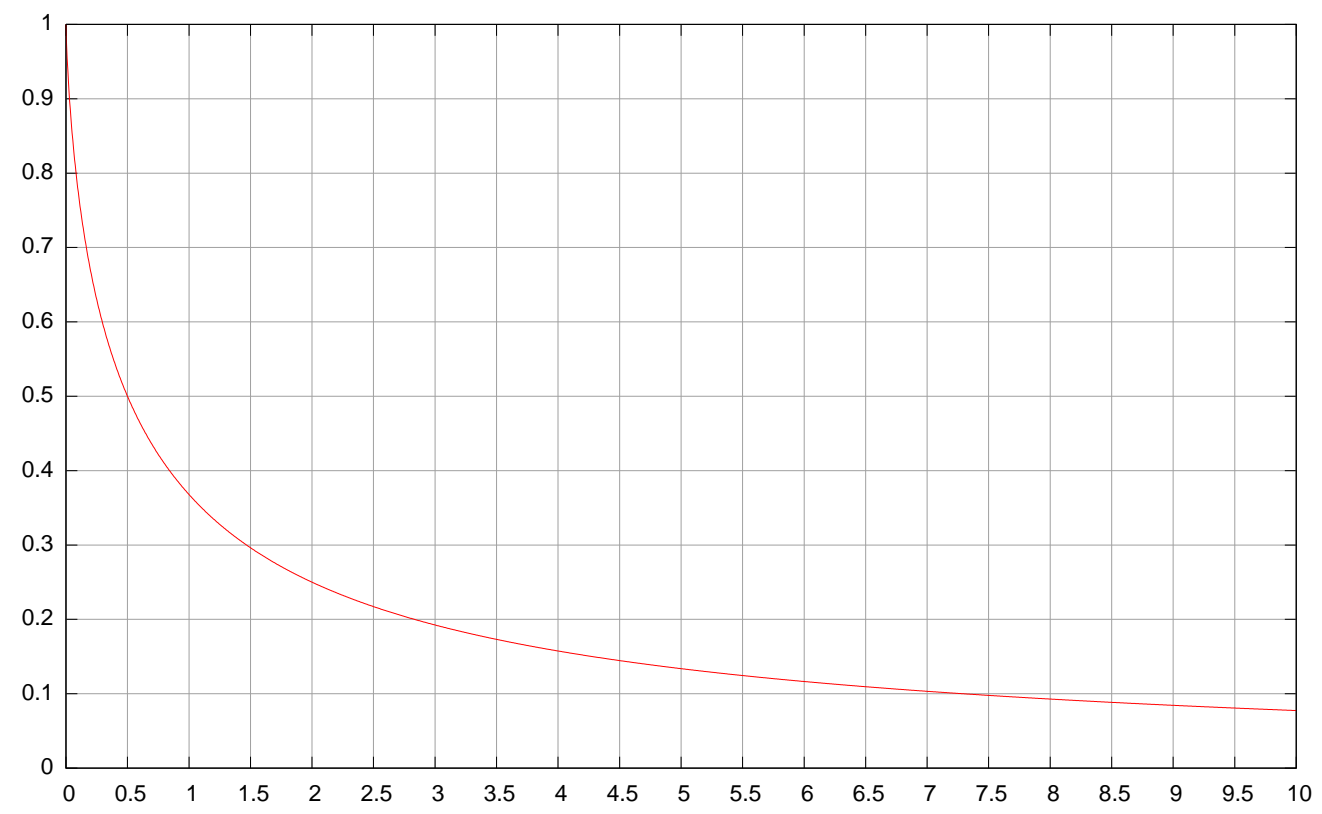

Fig. 1. Plot of $\rho^{\rho /(1-\rho)}$ vs. $0 \leq \rho \leq 10$; this is $C_{B, \max }$ as a function of $\rho=k_{2} / k_{1}$.

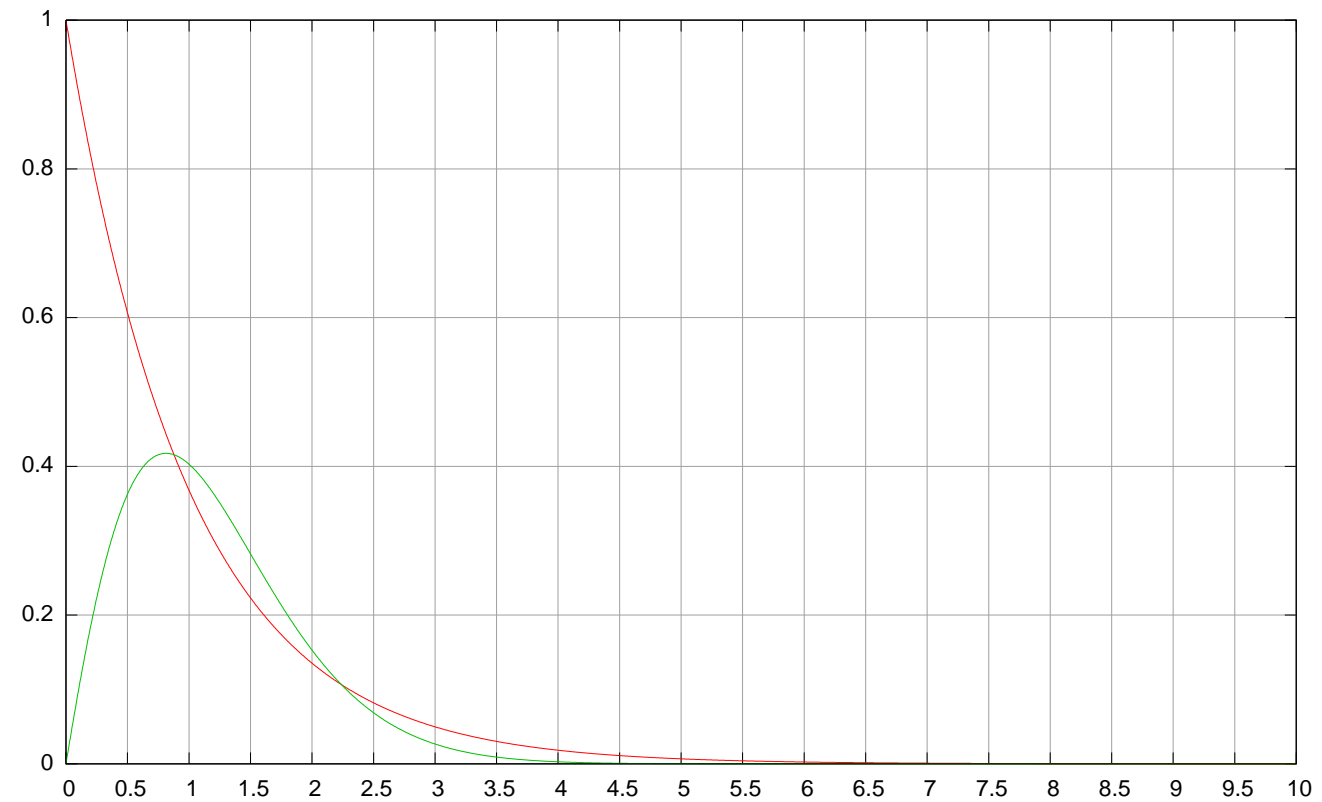

Fig. 2. Non-physical example of two non-osculating intersections. 


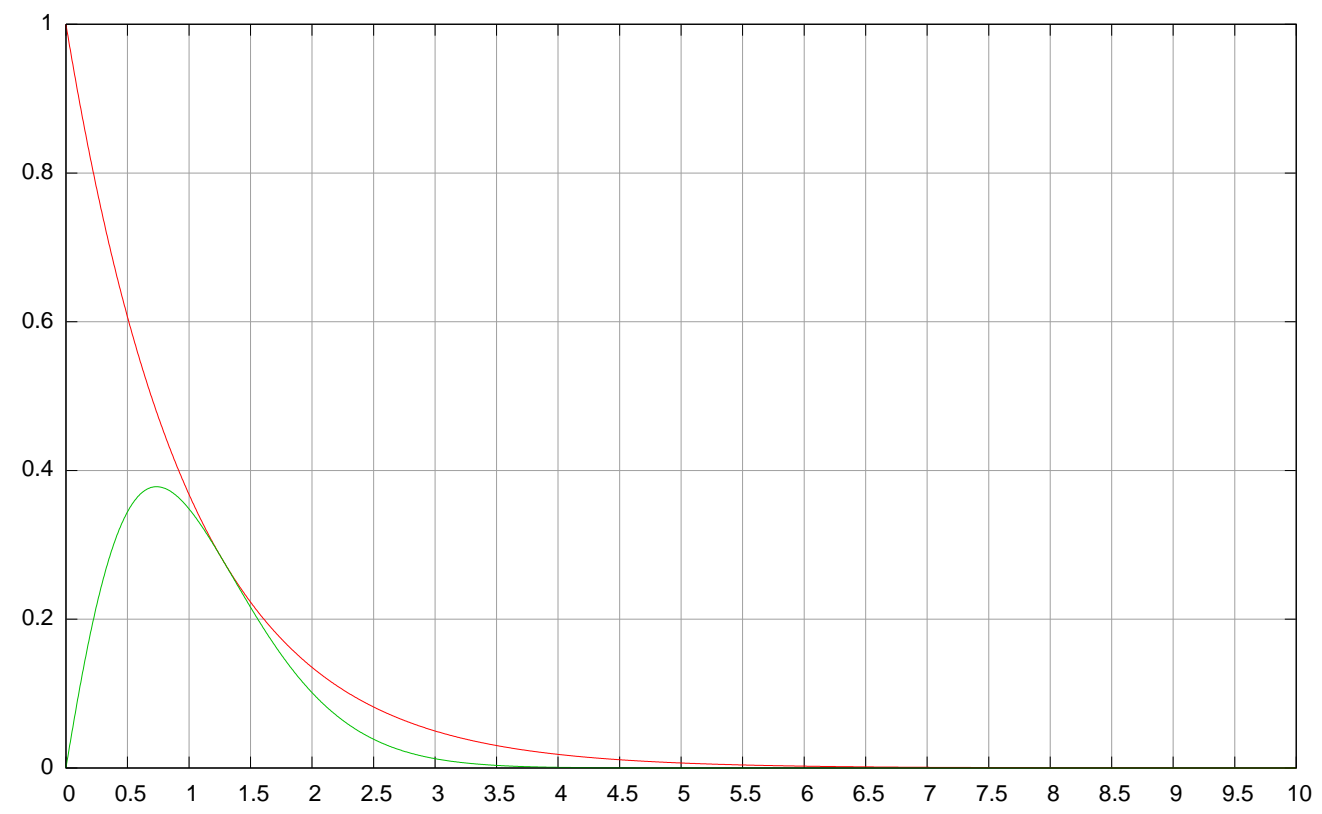

Fig. 3. Non-physical example of one finite osculating intersection.

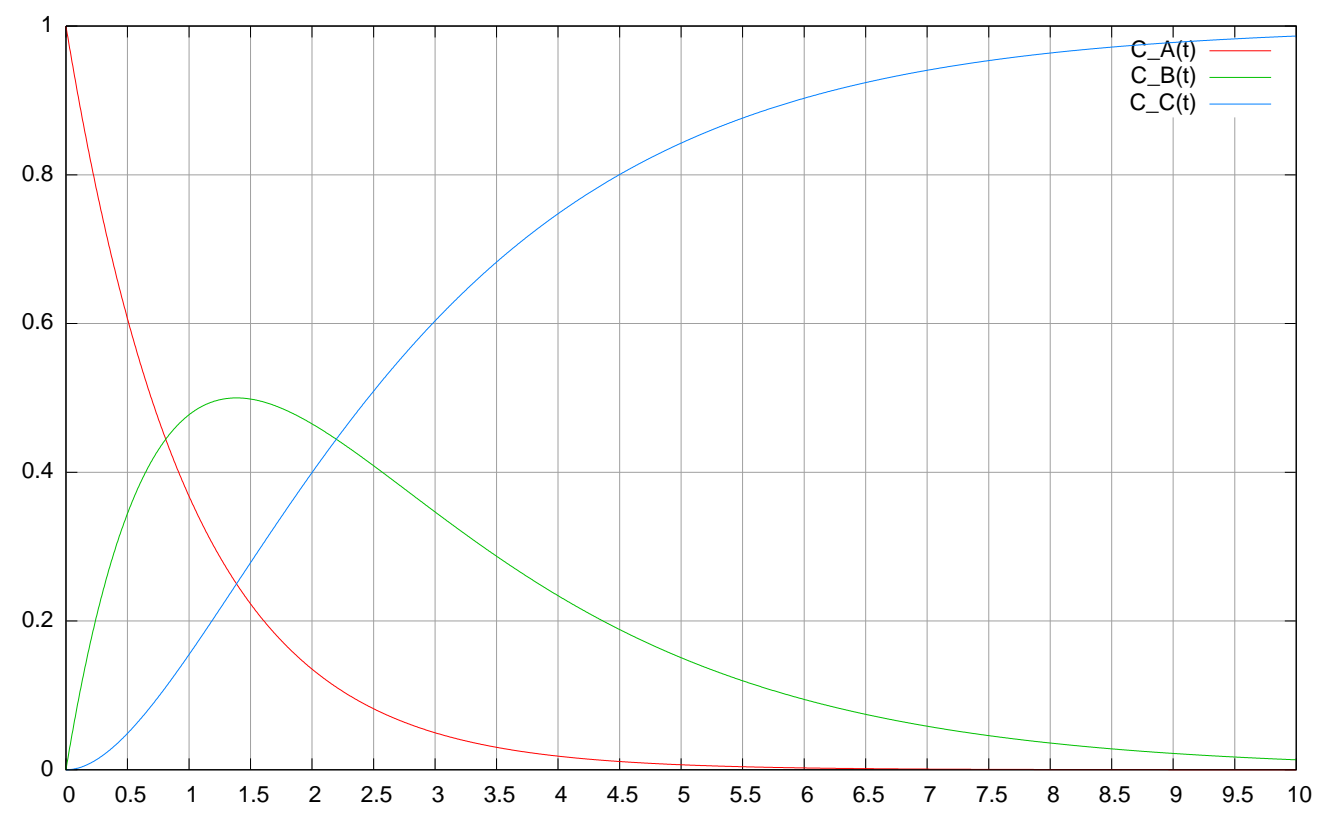

Fig. 4. Acme point $A$ : $k_{2}=0.5 \mathrm{~s}^{-1}, k_{1}=1 \mathrm{~s}^{-1}$. 


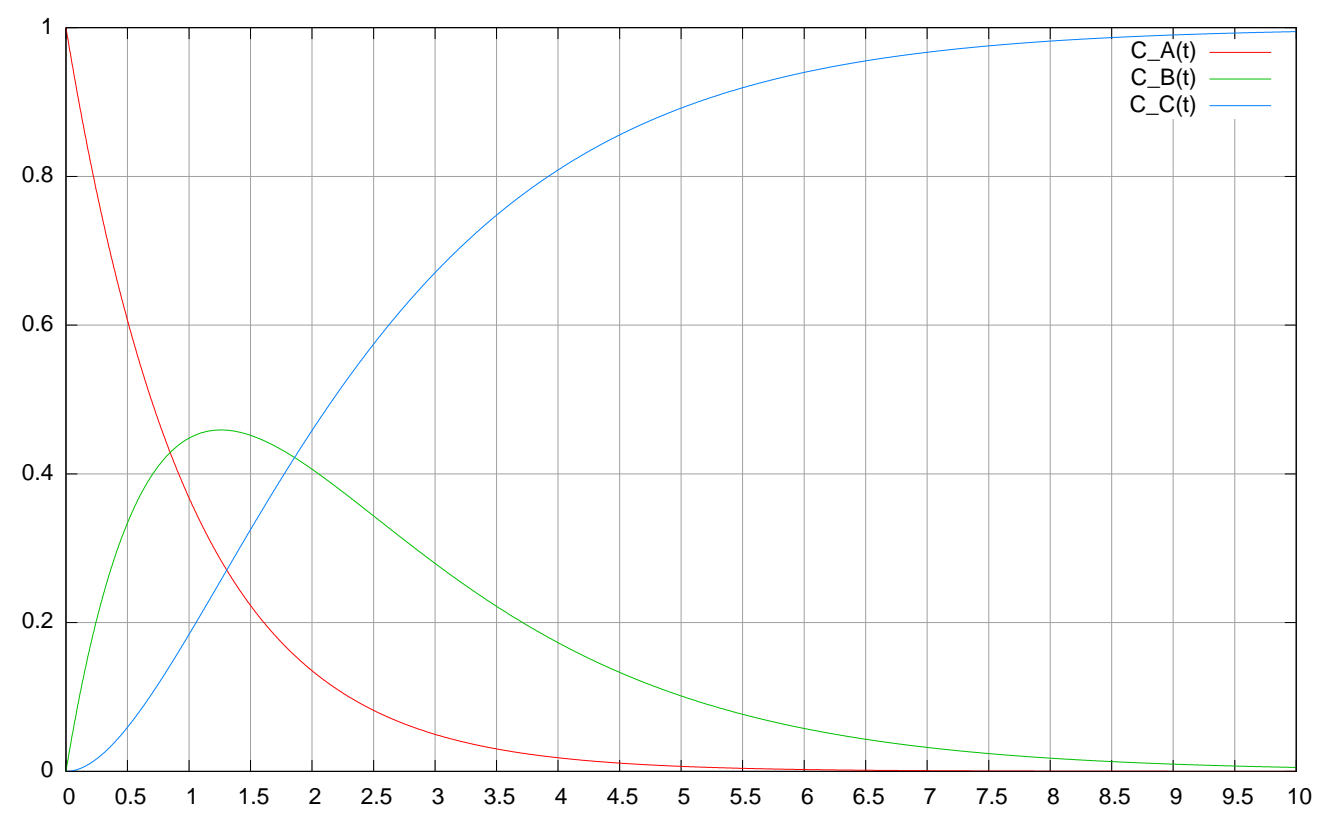

Fig. 5. Golden point $G$ : $k_{2}=(1 / \phi) \mathrm{s}^{-1}=0.618033, k_{1}=1 \mathrm{~s}^{-1}$.

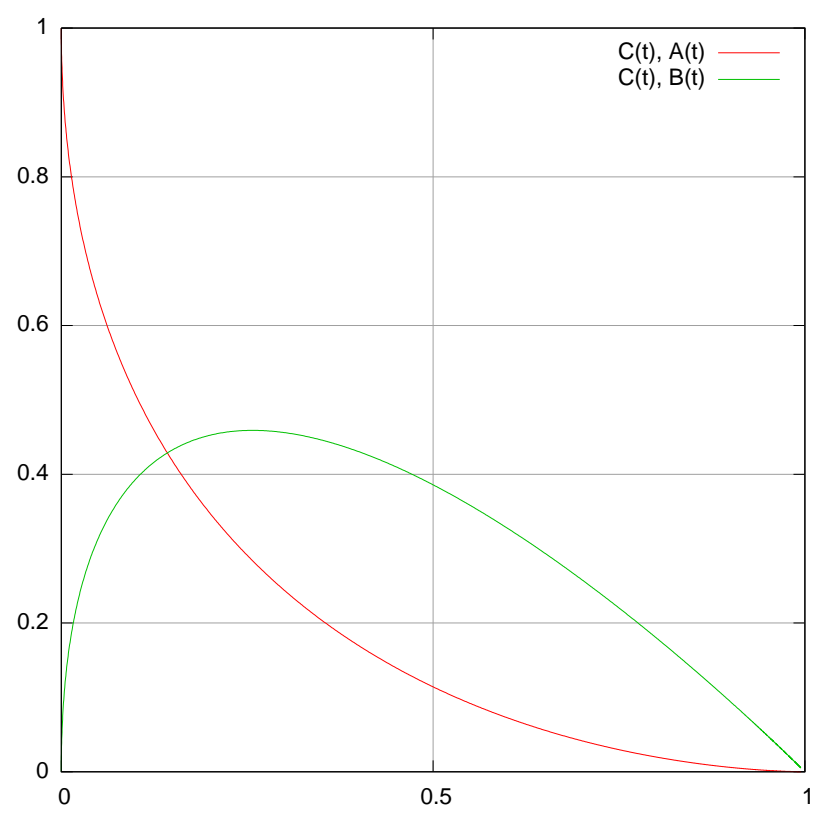

Fig. 6. Golden point $G$ : $k_{2}=(1 / \phi) \mathrm{s}^{-1}=0.618033, k_{1}=1 \mathrm{~s}^{-1}$. $A$ and $B$ vs. $C$. 


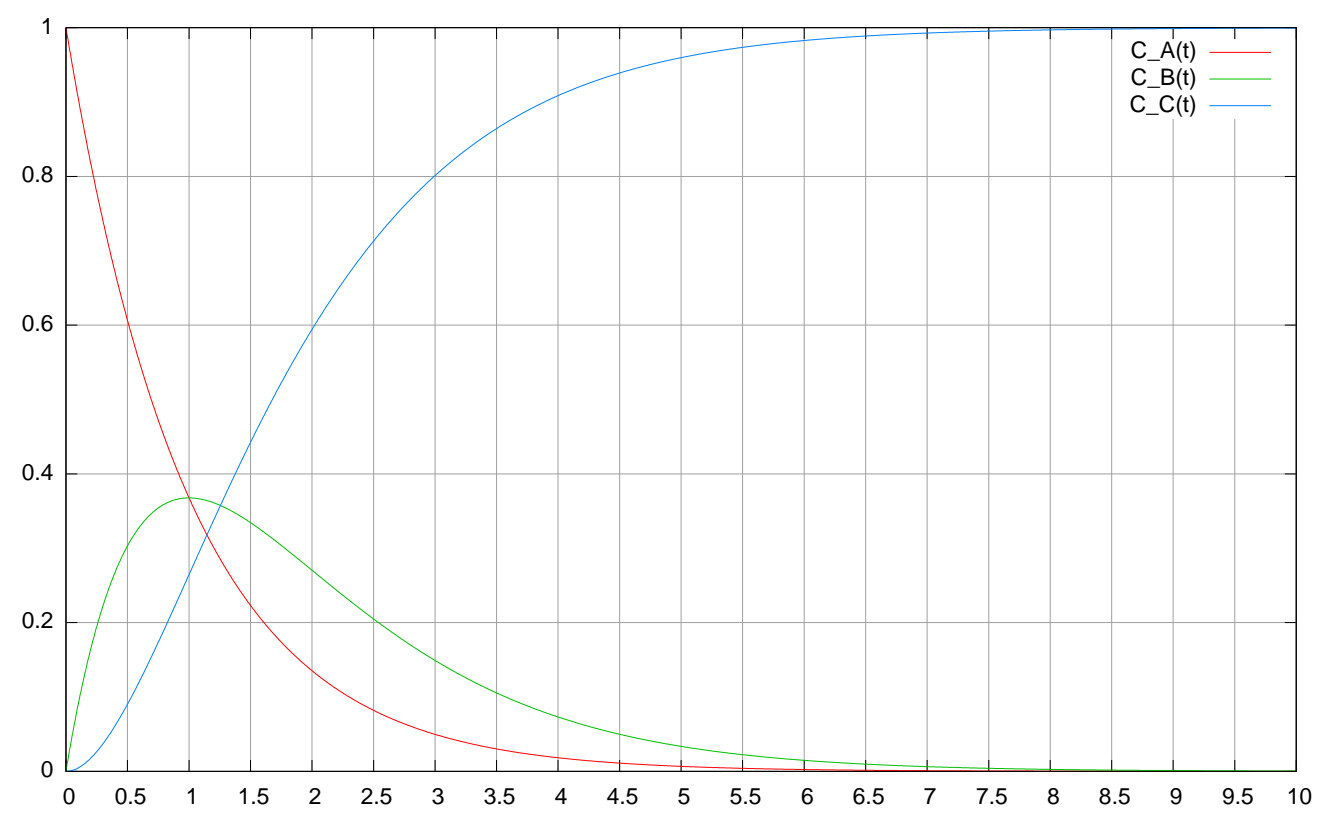

Fig. 7. Euler point $E: k_{2}=k_{1}=1 \mathrm{~s}^{-1}, t_{B, \max }=1 \mathrm{~s}$.

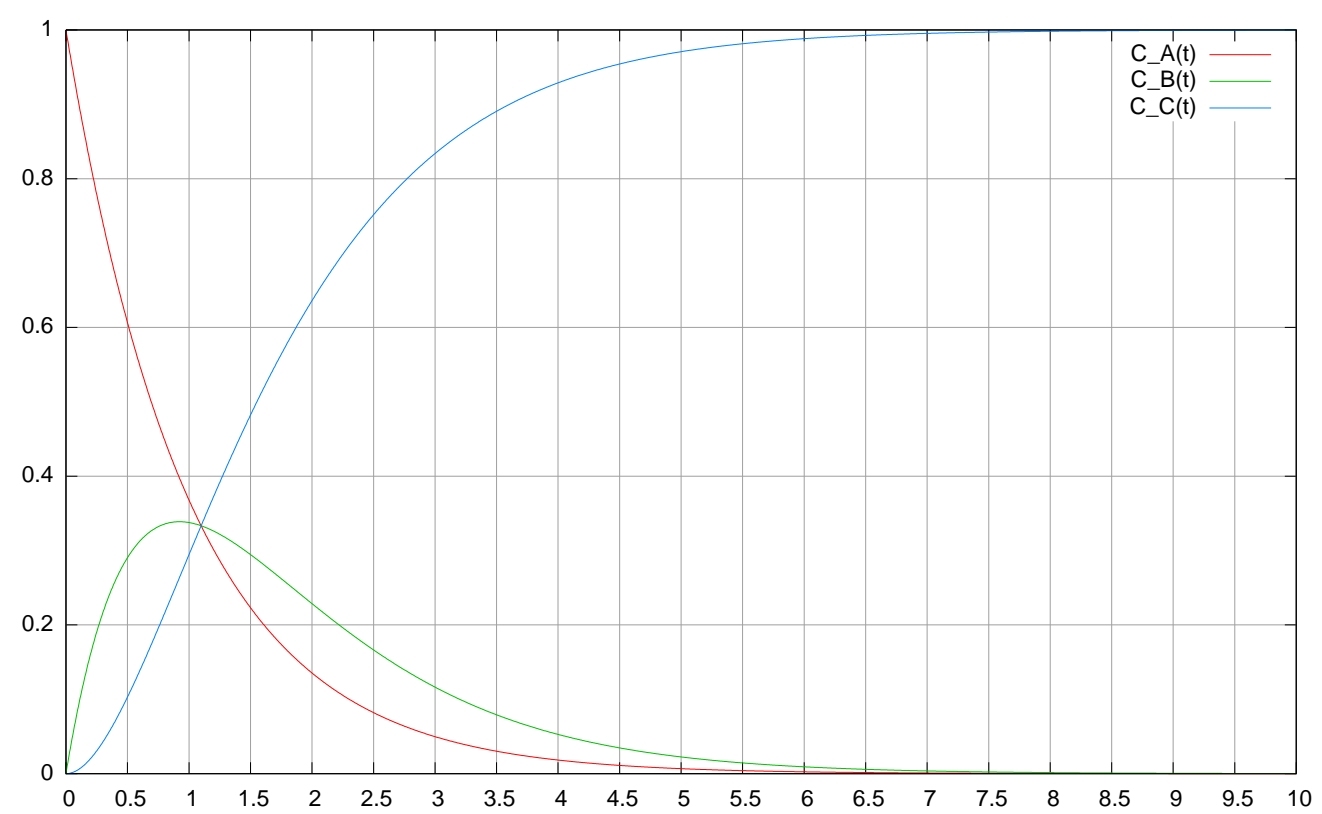

Fig. 8. Lambert point $L: k_{2}=1.1739824 \mathrm{~s}^{-1}, k_{1}=1 \mathrm{~s}^{-1}$. 


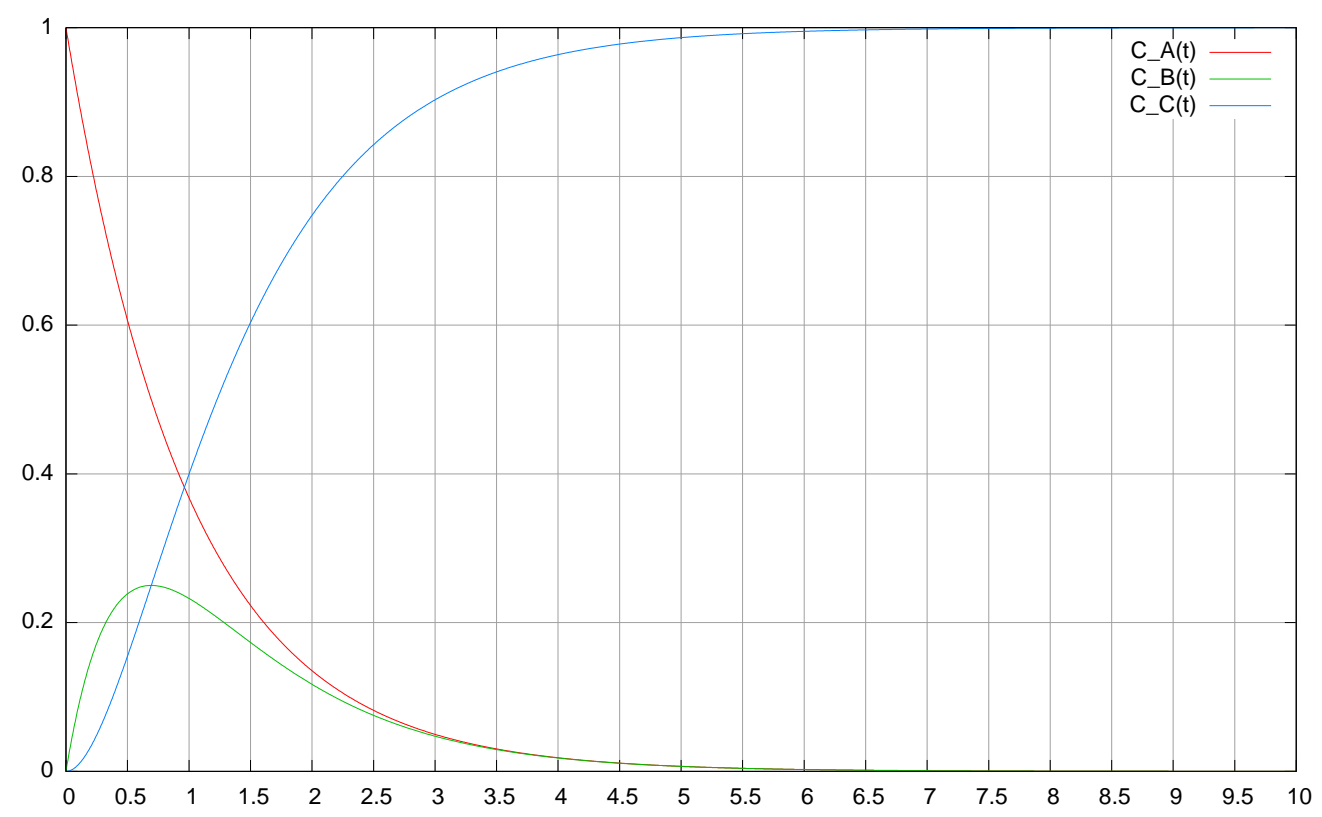

Fig. 9. Osculation point $O: k_{2}=2 \mathrm{~s}^{-1}, k_{1}=1 \mathrm{~s}^{-1}$.

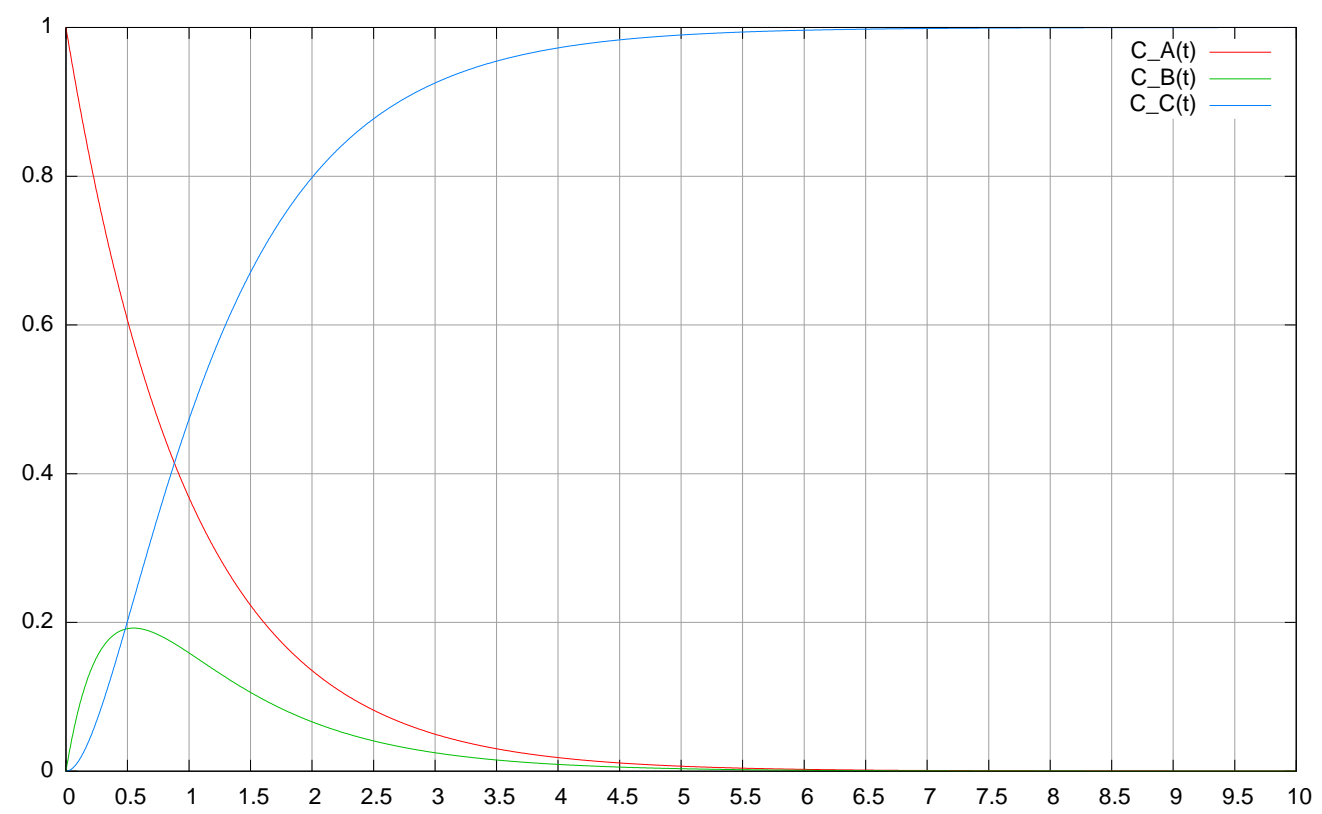

Fig. 10. Triad point $T: k_{2}=3 \mathrm{~s}^{-1}, k_{1}=1 \mathrm{~s}^{-1}$. 


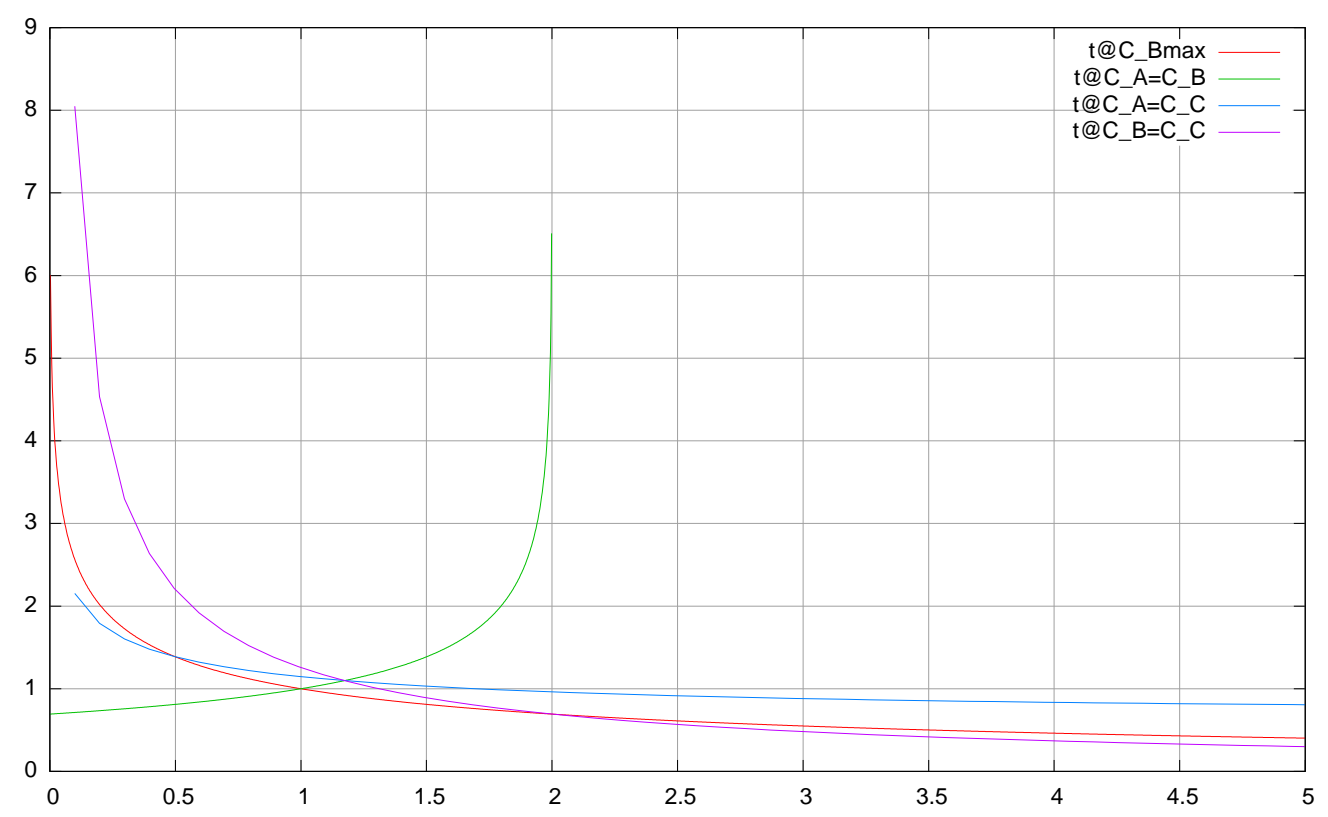

Fig. 11. Plot of times $t_{B, \max }$, intersection times of $C_{A}$ and $C_{B}$, then $C_{A}$ and $C_{C}$, then $C_{B}$ and $C_{C}$

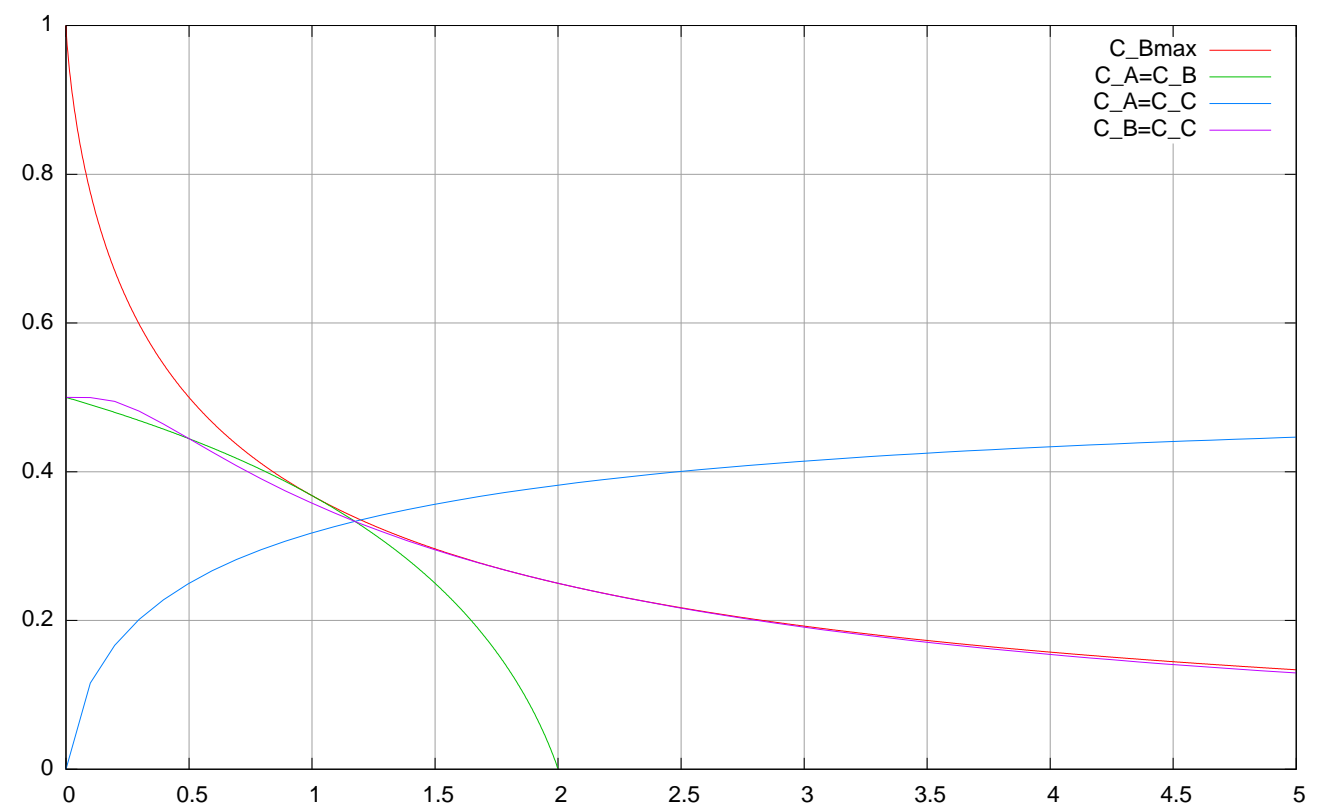

Fig. 12. Plot of $C_{B, \max }$, intersections of $C_{A}$ and $C_{B}$, then $C_{A}$ and $C_{C}$, then $C_{B}$ and $C_{C}$ 


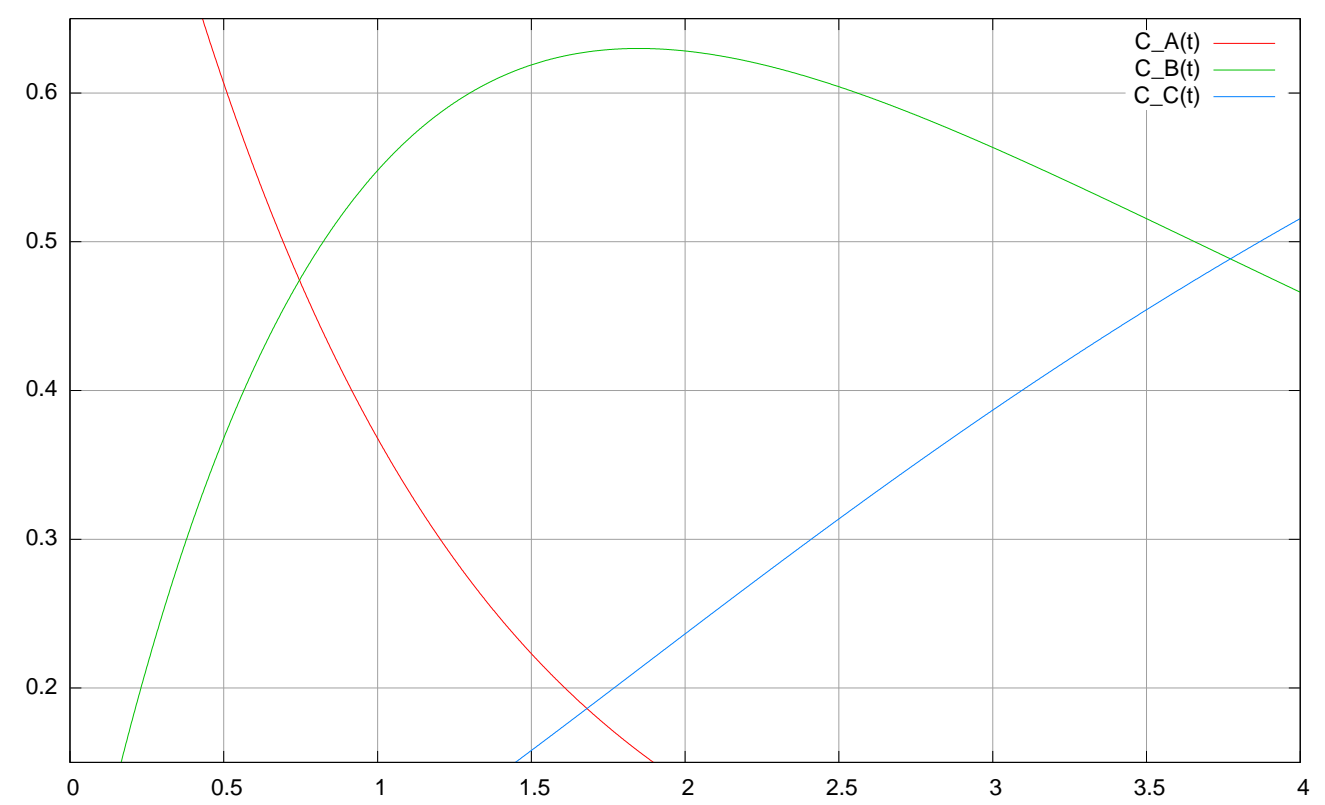

Fig. 13. Example (detail) of domain D1: plot of $C_{A}, C_{B}$ and $C_{C}$ vs. time for $k_{2}=0.25 \mathrm{~s}^{-1}, k_{1}=1 \mathrm{~s}^{-1}$. In time, $\mathrm{AB}<\mathrm{AC}<\mathrm{Bm}<\mathrm{BC}$; in value, $\mathrm{AC}<\mathrm{AB}<\mathrm{BC}<\mathrm{Bm}$.

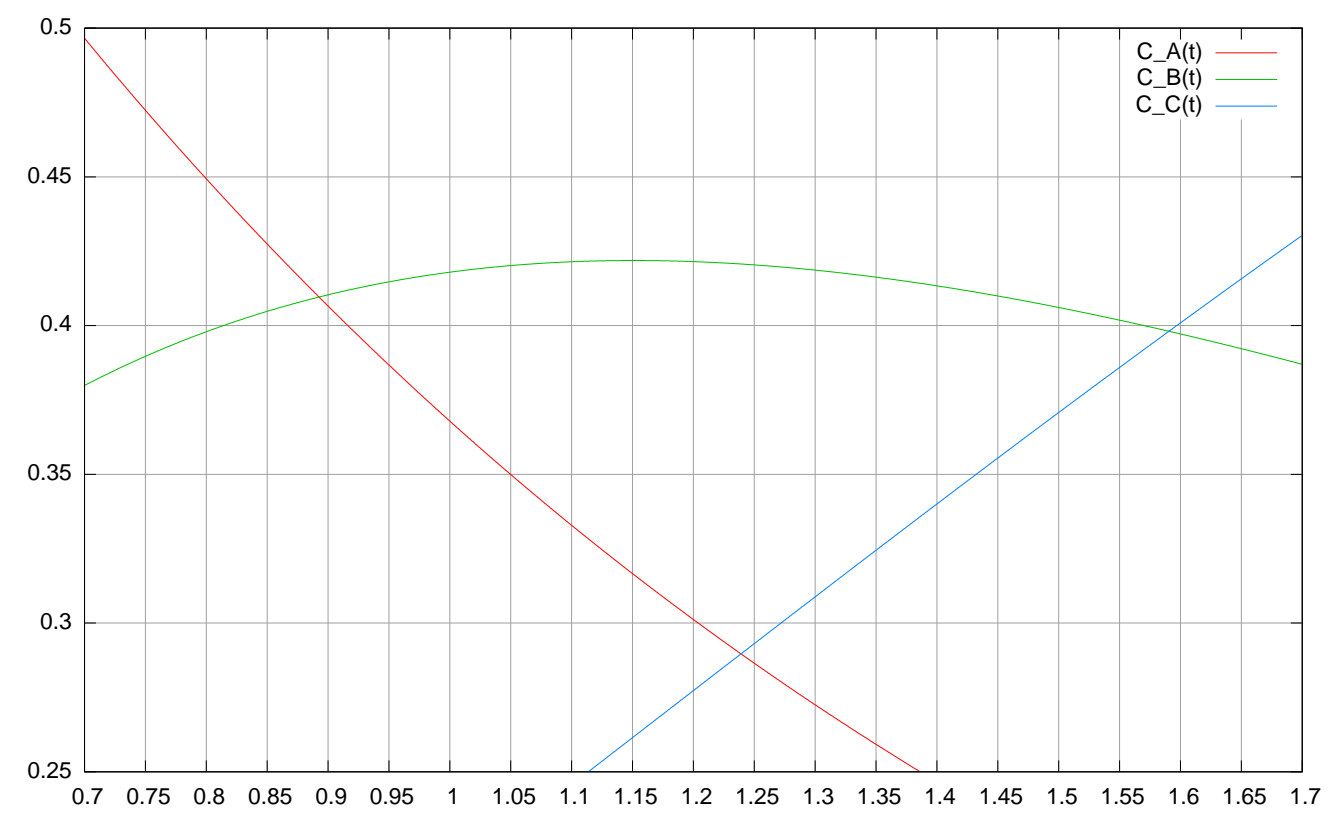

Fig. 14. Example (detail) of domain D2: plot of $C_{A}, C_{B}$ and $C_{C}$ vs. time for $k_{2}=0.75 \mathrm{~s}^{-1}, k_{1}=1 \mathrm{~s}^{-1}$. In time, $\mathrm{AB}<\mathrm{Bm}<\mathrm{AC}<\mathrm{BC}$; in value, $\mathrm{AC}<\mathrm{BC}<\mathrm{AB}<\mathrm{Bm}$. 


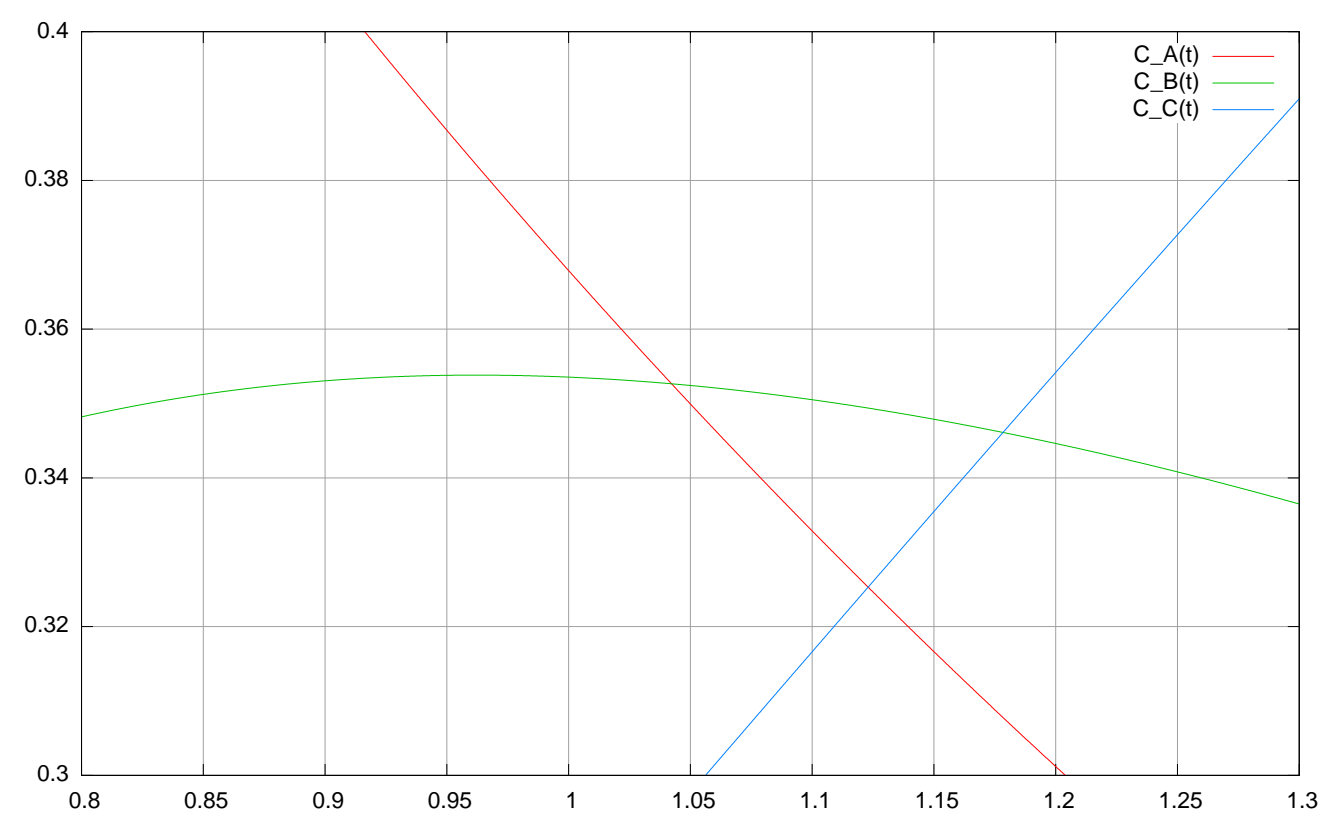

Fig. 15. Example (detail) of domain D3: plot of $C_{A}, C_{B}$ and $C_{C}$ vs. time for $k_{2}=1.08 \mathrm{~s}^{-1}, k_{1}=1 \mathrm{~s}^{-1}$. In time, $\mathrm{Bm}<\mathrm{AB}<\mathrm{AC}<\mathrm{BC}$; in value, $\mathrm{AC}<\mathrm{BC}<\mathrm{AB}<\mathrm{Bm}$.

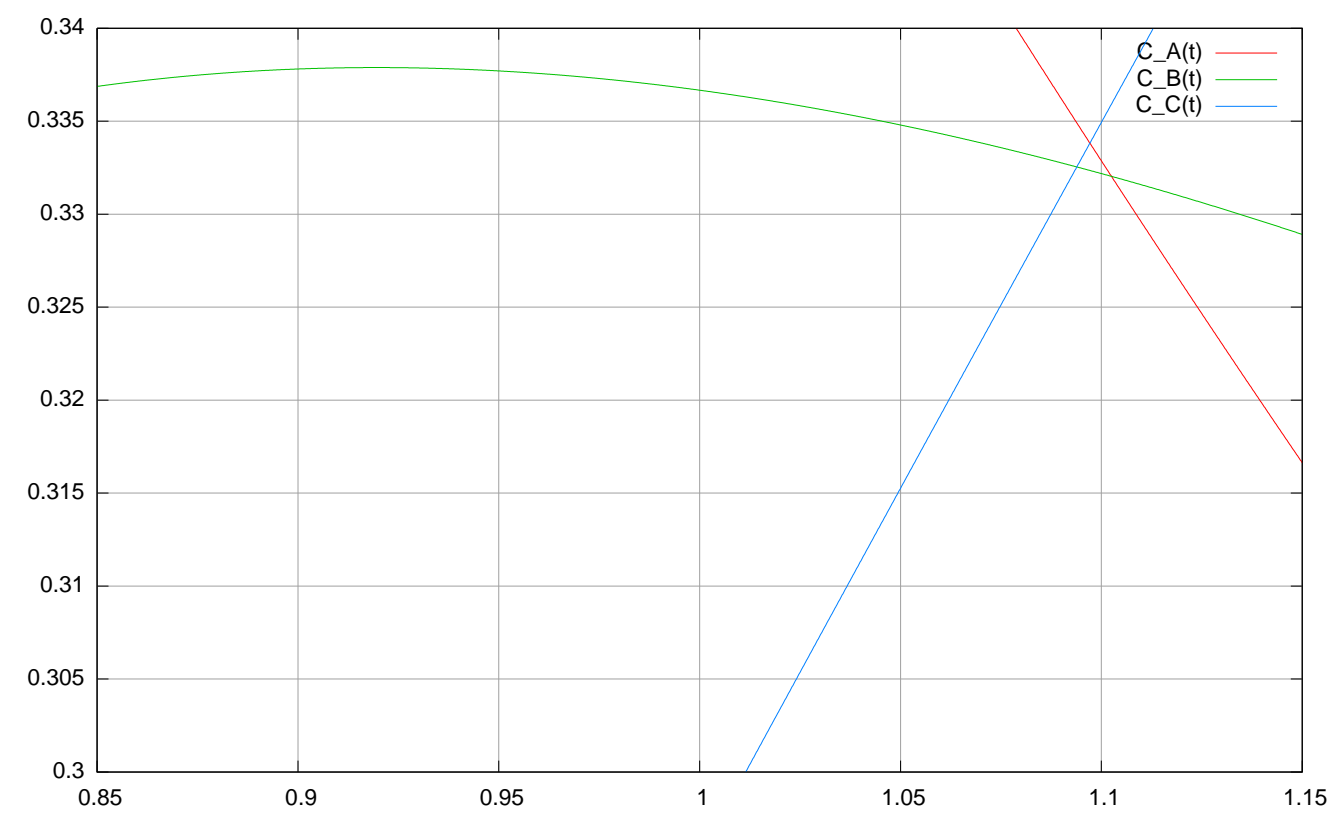

Fig. 16. Example (detail) of domain D4a: plot of $C_{A}, C_{B}$ and $C_{C}$ vs. time for $k_{2}=1.18 \mathrm{~s}^{-1}, k_{1}=1 \mathrm{~s}^{-1}$. In time, $\mathrm{Bm}<\mathrm{BC}<\mathrm{AC}<\mathrm{AB}$; in value, $\mathrm{AB}<\mathrm{BC}<\mathrm{AC}<\mathrm{Bm}$.

\section{A Positivity of a certain expression and the monotone increasing natural logarithm}

Any value of the form $\ln (x / y) /(x-y)$, with $x>0, y>0, x \neq y$, is positive: it can be rewritten as $(\ln x-\ln y) /(x-y)$, and since the $\ln$ is monotone 


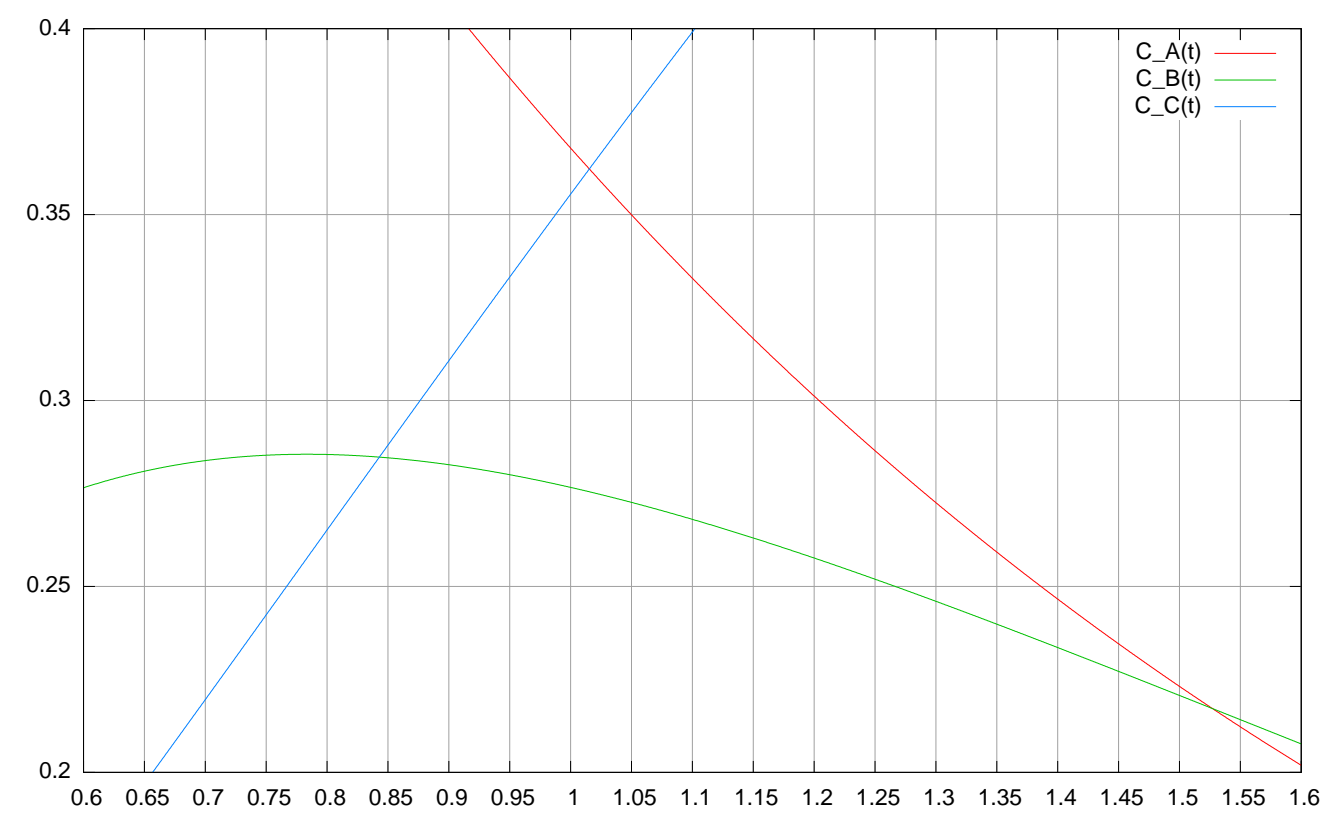

Fig. 17. Example (detail) of domain D4b: plot of $C_{A}, C_{B}$ and $C_{C}$ vs. time for $k_{2}=1.6 \mathrm{~s}^{-1}, k_{1}=1 \mathrm{~s}^{-1}$. In time, $\mathrm{Bm}<\mathrm{BC}<\mathrm{AC}<\mathrm{AB}$; in value, $\mathrm{AB}<\mathrm{BC}<\mathrm{Bm}<\mathrm{AC}$.

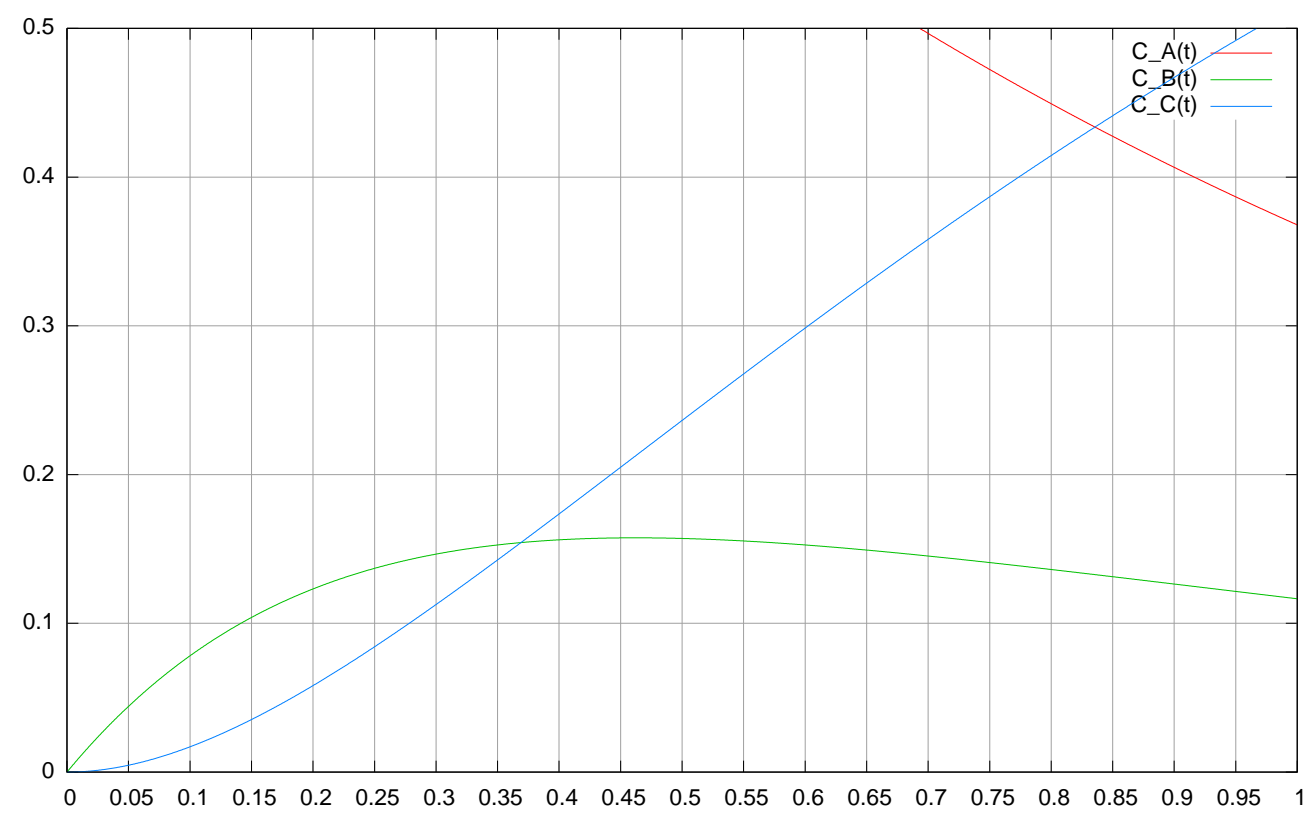

Fig. 18. Example (detail) of domain D5: plot of $C_{A}, C_{B}$ and $C_{C}$ vs. time for $k_{2}=4 \mathrm{~s}^{-1}, k_{1}=1 \mathrm{~s}^{-1}$. In time, $\mathrm{BC}<\mathrm{Bm}<\mathrm{AC}$; in value, $\mathrm{BC}<\mathrm{Bm}<\mathrm{AC}$, no $\mathrm{AB}$.

increasing, the sign of the numerator is the same as that of the denominator, so the quotient is positive. 


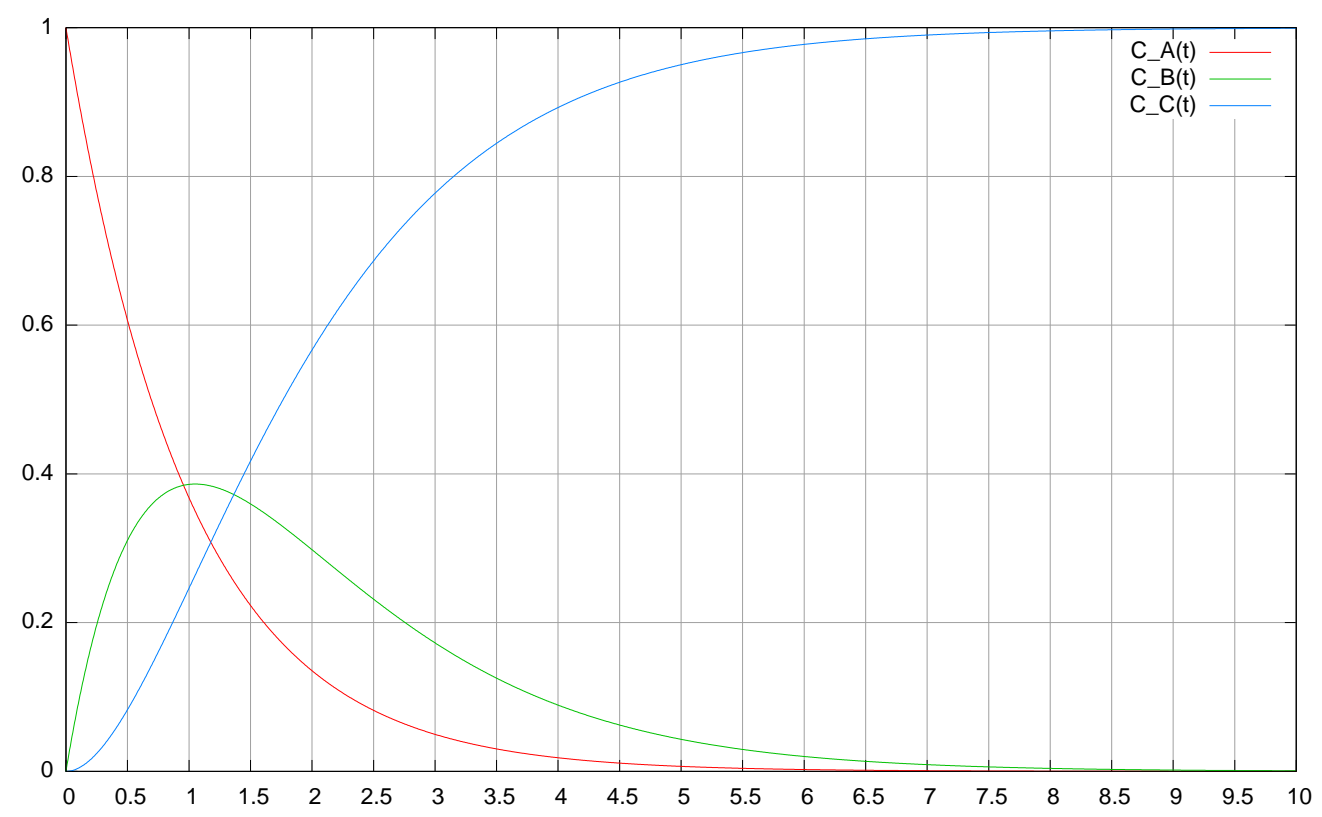

Fig. 19. Plot of $C_{A}, C_{B}$ and $C_{C}$ vs. time for $k_{2} / k_{1}=3.371 / 3.724=0.9052$, close to Euler point $E$

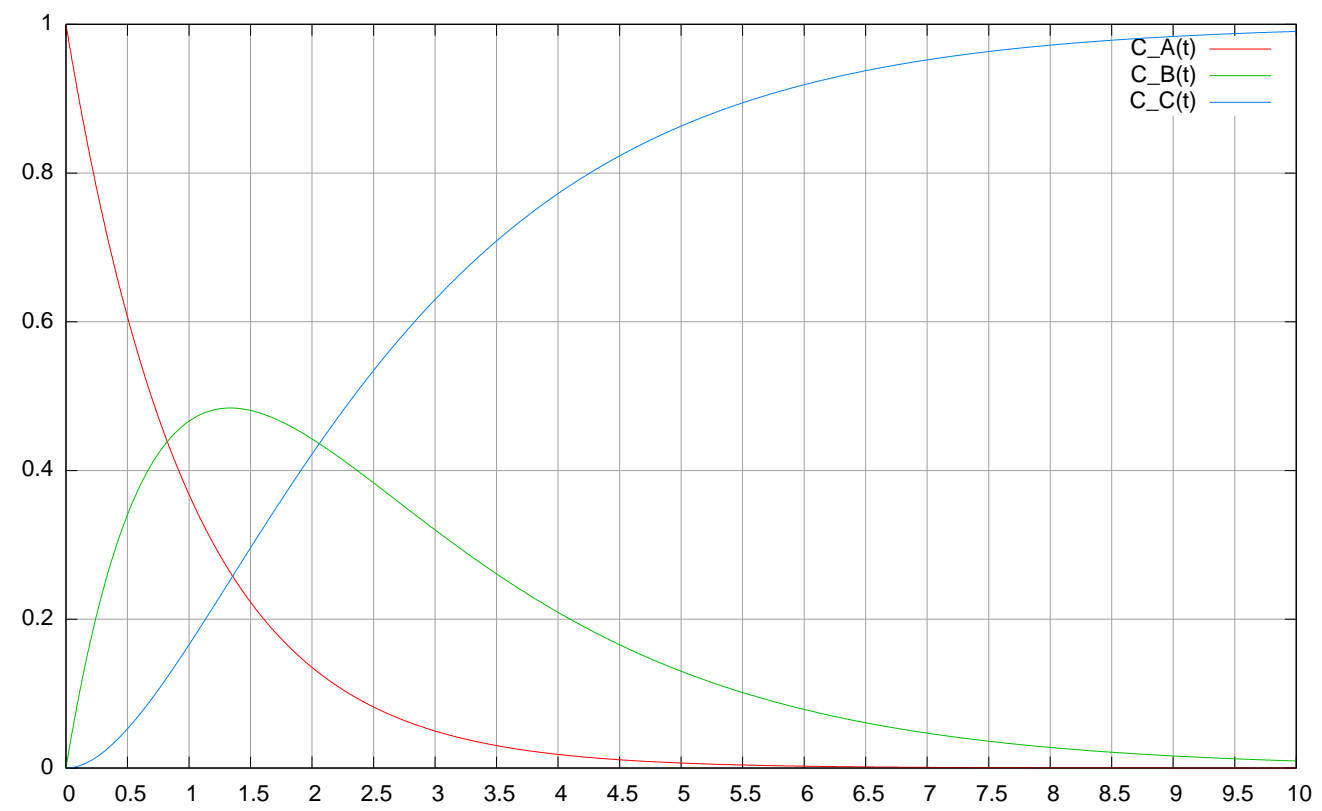

Fig. 20. Plot of $C_{A}, C_{B}$ and $C_{C}$ vs. time for $k_{2} / k_{1}=5.616 / 10.344=0.5429$, close to Acme point $A$

\section{B Lambert $W$ function}

Any solution $x$ to $x e^{x}=y$ is a value of the Lambert function $W(y)$. This definition creates an infinite number of branches of complex functions; by $W_{0}$ we denote the so-called principal branch, which is real on both sides near 


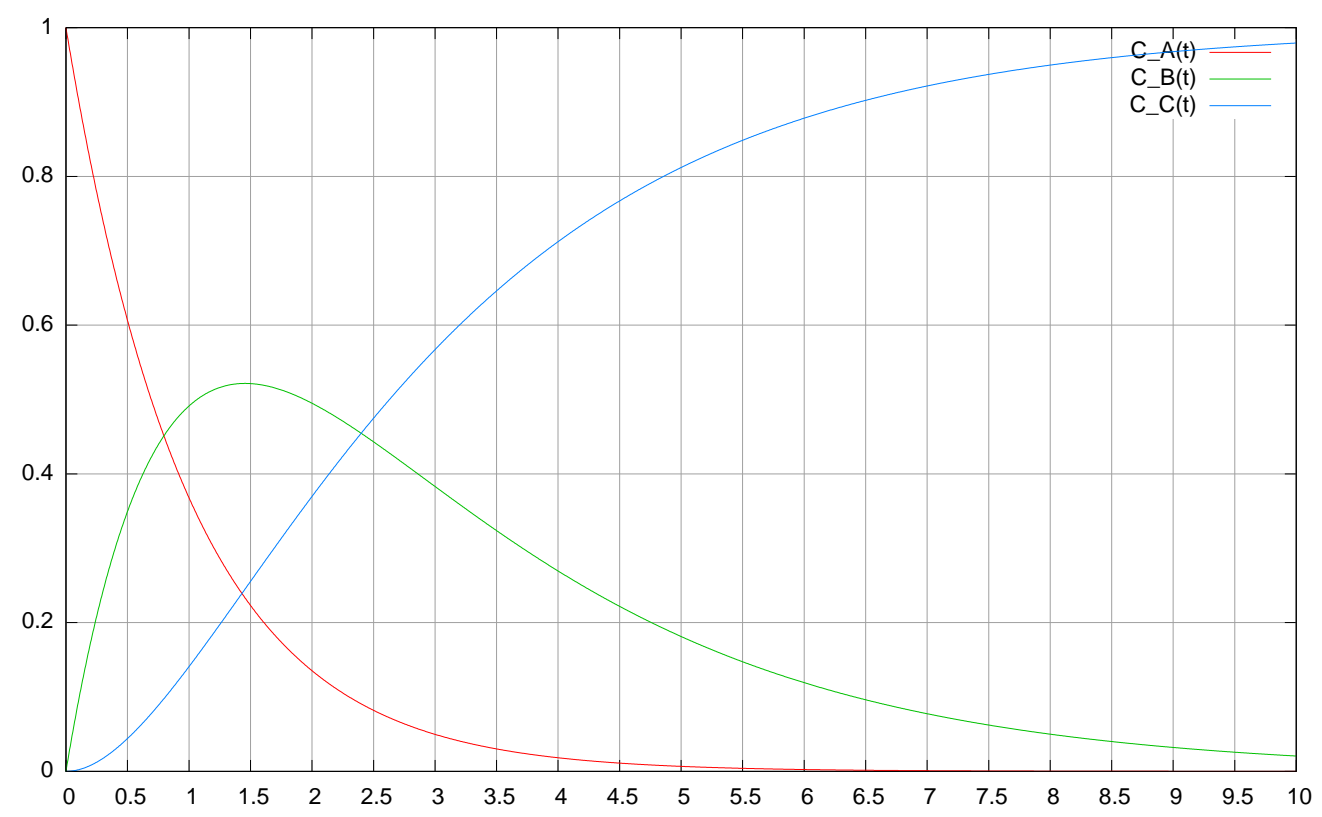

Fig. 21. Plot of $C_{A}, C_{B}$ and $C_{C}$ vs. time for $k_{2} / k_{1}=4.623 / 10.344=0.4469$, close to Acme point $A$

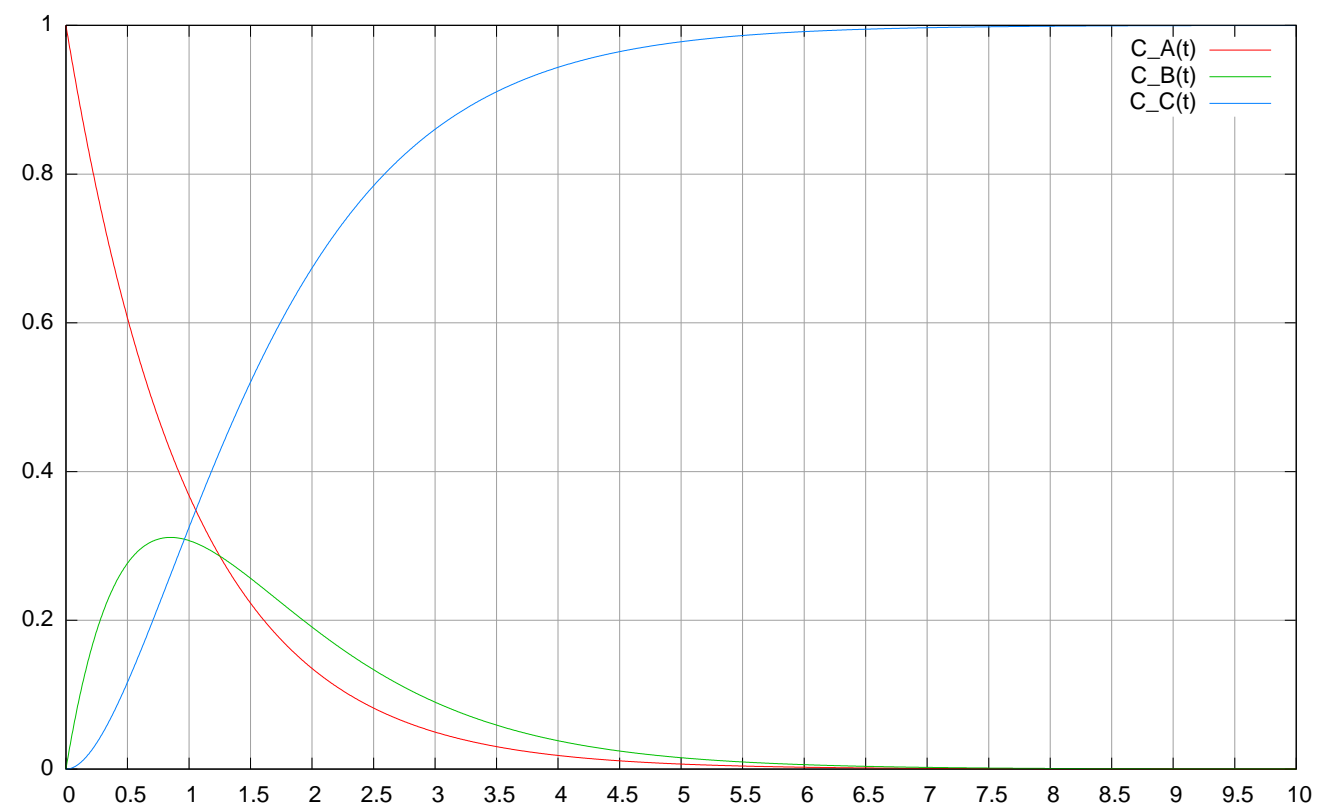

Fig. 22. Plot of $C_{A}, C_{B}$ and $C_{C}$ vs. time for $k_{2} / k_{1}=4.623 / 3.371=1.3714$, not close to any special point, between $L$ and $O$.

$y=0$, and by $W_{-1}$ the branch that is real from $y=-1 / e$ to 0 (but no further). See Fig. C.1 and (Corless et al., 1996). 


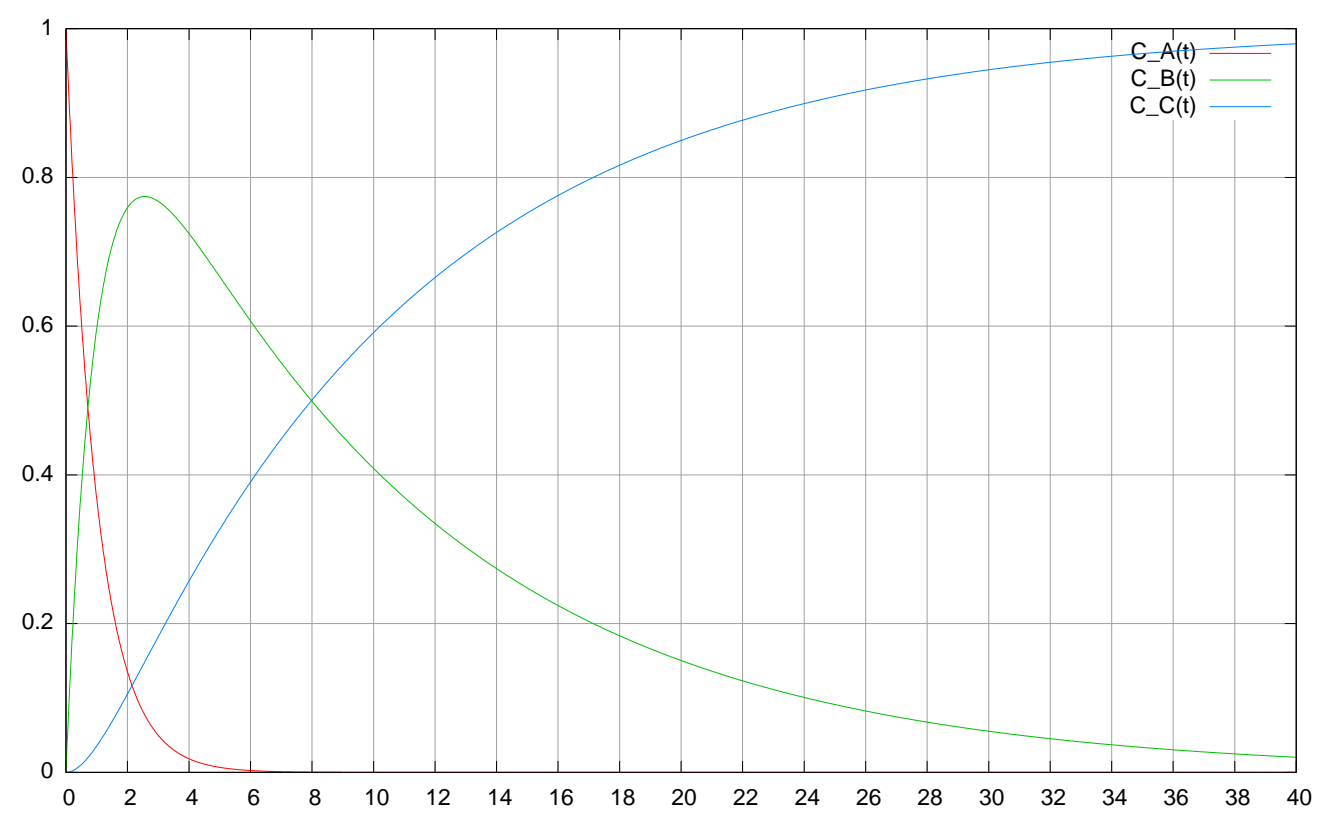

Fig. 23. Large $k_{1}$ example: $k_{2}=0.1 \mathrm{~s}^{-1}, k_{1}=1 \mathrm{~s}^{-1}$.

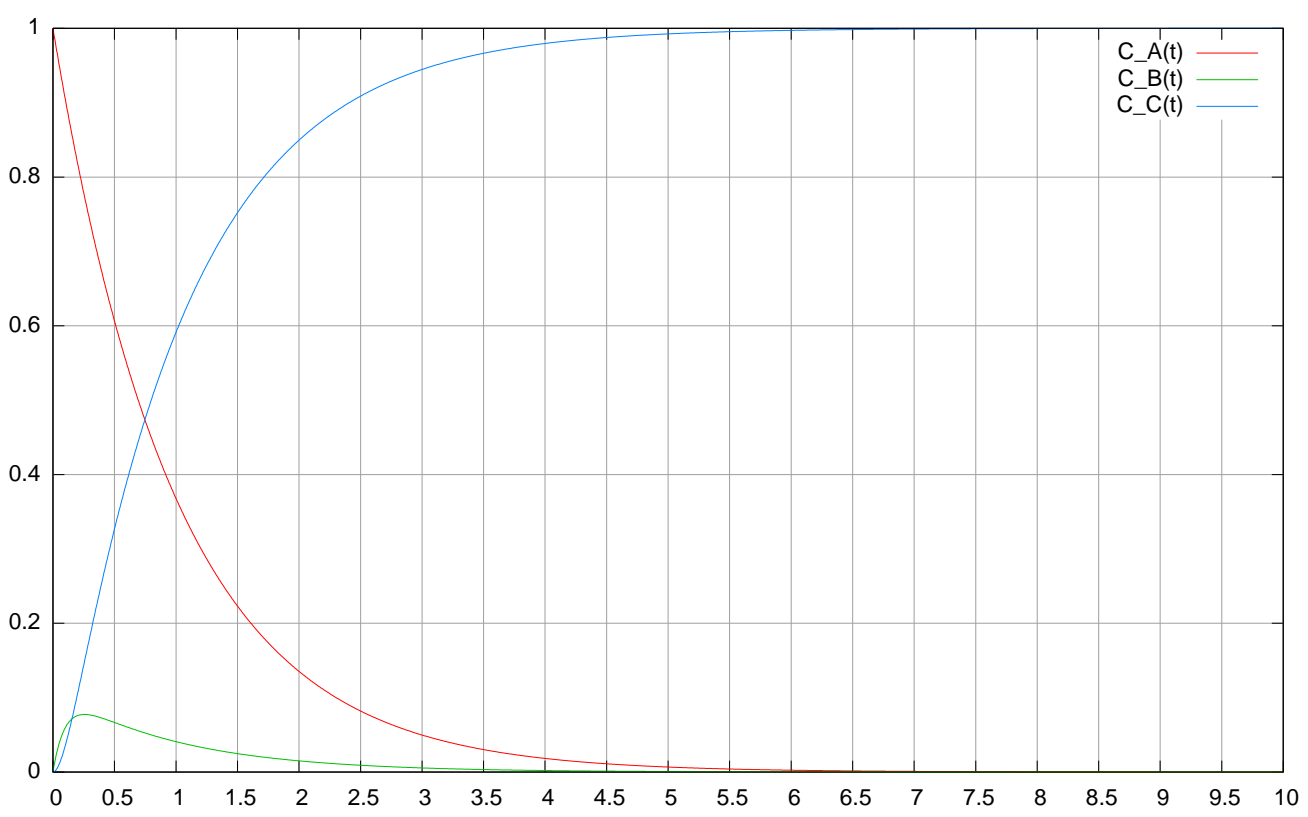

Fig. 24. Large $k_{2}$ example: $k_{2}=10 \mathrm{~s}^{-1}, k_{1}=1 \mathrm{~s}^{-1}$.

C Laplace-domain solution of (1)-(3)

Applying the Laplace transform, the system becomes

$$
\begin{aligned}
& s \mathcal{L} C_{A}-C_{A, 0}=-k_{1} \mathcal{L} C_{A}, \\
& s \mathcal{L} C_{B}-C_{B, 0}=k_{1} \mathcal{L} C_{A}-k_{2} \mathcal{L} C_{B},
\end{aligned}
$$




$$
s \mathcal{L} C_{C}=k_{2} \mathcal{L} C_{B},
$$

whence by elementary manipulations,

$$
\begin{aligned}
\mathcal{L} C_{A} & =\frac{C_{A, 0}}{s+k_{1}}, \\
\mathcal{L} C_{B} & =\frac{k_{1} C_{A, 0}}{\left(s+k_{1}\right)\left(s+k_{2}\right)}+\frac{C_{B, 0}}{s+k_{2}}, \\
\mathcal{L} C_{C} & =\frac{k_{1} k_{2} C_{A, 0}}{s\left(s+k_{1}\right)\left(s+k_{2}\right)}+\frac{k_{2} C_{B, 0}}{s\left(s+k_{2}\right)} .
\end{aligned}
$$

The case $k_{1}=k_{2}$ only has to be distinguished from the general case at this point because it causes a double pole to occur in the partial fraction expansions of the first terms on the right-hand sides of (C.5) and (C.6).

\section{References}

Bairamov, V.M. Osnovy chimicheskoj kinetiki i kataliza (in Russian, Foundations of Chemical Kinetics and Catalysis), textbook for Chemical Schools of Russian Universities, ed. Acad. V.V. Lunin. Moscow: Academa, 254pp, 2003.

Corless, R.M., Gonnet, G.H., Hare, D.E.G., Jeffrey, D.J., and Knuth, D.E. On the Lambert W Function, Advances in Computational Mathematics Vol. 5, 329-359, 1996.

Eremin, E.N. Osnovy chimicheskoj kinetiki. Moscow: University Vysshaya Shkola, 1976.

Froment, G.F. and Bischoff, K.B. Chemical Reactor Analysis and Design, Wiley Series in Chemical Engineering, 1990.

Kubasov, A.A. Chemical Kinetics and Catalysis I. Statistical equilibrium phenomenological kinetics. http://www.chemnet.ru/rus/teaching/kubasov/02.pdf. Based on the textbook: Kubasov, A.A. Chemical Kinetics and Catalysis I. Moscow: Moscow University Publishing, 2004.

Hlavacek, V., Puszinski, J.A., Viljoen, H.J, Gatica, J.E. Model Reactors and Their Design Equations, Ullman's Encyclopedia of Industrial Chemistry, Wiley, 2007.

Lederer, C.M., Hollander, J. M., Perlman, I. Table of Isotopes, 6th ed. Wiley and Sons: New York 1968.

Wei, J., Prater, C.D. Structure and analysis of complex reaction systems. Advances in Catalysis 13 (203), 202-392, 1962. 
List of figure captions (in the Appendices)

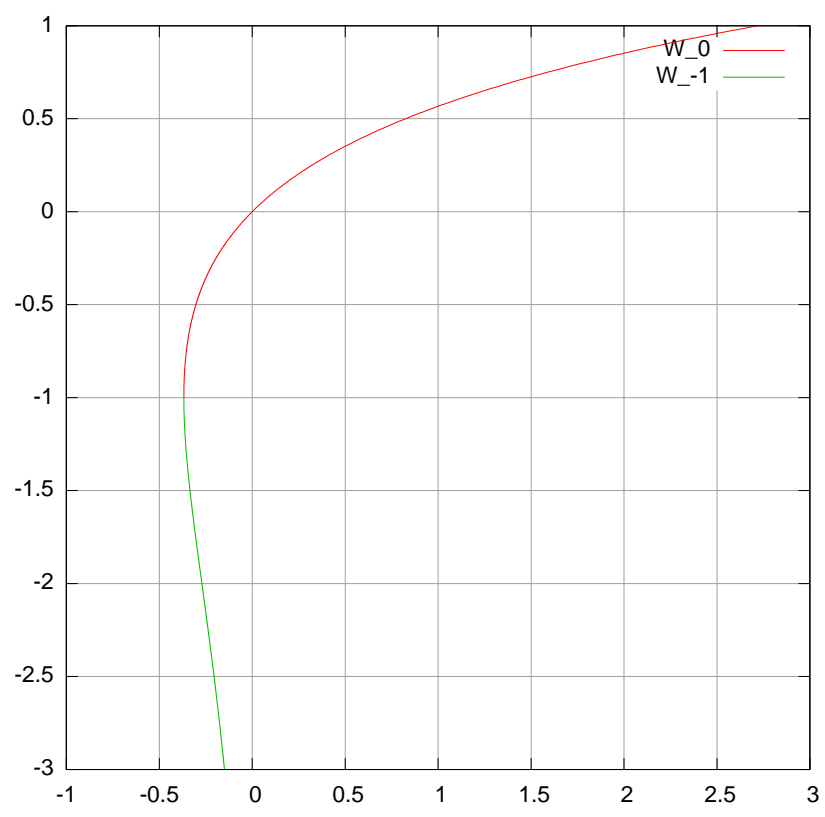

Fig. C.1. The branches $W_{0}$ and $W_{-1}$ of the Lambert $W$ function. 